九州大学学術情報リポジトリ

Kyushu University Institutional Repository

生後早期のアレルギー性気道炎症はジストロフィー 型ミクログリアを誘導し，興奮性後シナプス余剰と 自閉症様行動を喚起する

齋藤，万有

http://hdl. handle. net/2324/4496012

出版情報: Kyushu University，2021，博士（医学），課程博士 バージョン:

権利関係: (c)2021 Elsevier Inc. All rights reserved. 


\title{
Early postnatal allergic airway inflammation induces dystrophic microglia leading to excitatory postsynaptic surplus and autism-like behavior
}

\author{
Ban-yu Saitoh $^{\mathrm{a}}$, Eizo Tanaka ${ }^{\mathrm{a}}$, Norio Yamamoto ${ }^{\mathrm{b}}$, Daan van Kruining ${ }^{\mathrm{c}}$, Kyoko Iinuma ${ }^{\mathrm{a}}$, \\ Yuko Nakamuta ${ }^{a}$, Hiroo Yamaguchi ${ }^{\mathrm{a}}$, Ryo Yamasaki ${ }^{\mathrm{a}}$, Koichiro Matsumoto ${ }^{\mathrm{b}}$, \\ Jun-ichi Kira ${ }^{a, d, e, *}$ \\ ${ }^{a}$ Department of Neurology, Neurological Institute, Graduate School of Medical Sciences, Kyushu University, Fukuoka, Japan \\ ${ }^{\mathrm{b}}$ Research Institute for Diseases of the Chest, Graduate School of Medical Sciences, Kyushu University, Fukuoka, Japan \\ ${ }^{\mathrm{c}}$ School for Mental Health and Neuroscience, Department of Psychiatry and Neuropsychology, Maastricht University, Universiteitssingel 50, 6229 ER Maastricht, \\ Netherlands \\ ${ }^{\mathrm{d}}$ Translational Neuroscience Center, Graduate School of Medicine, and School of Pharmacy at Fukuoka, International University of Health and Welfare, 137-1 Enokizu, \\ Ookawa, Fukuoka 831-8501, Japan \\ e Department of Neurology, Brain and Nerve Center, Fukuoka Central Hospital, International University of Health and Welfare, 2-6-11 Yakuin, Chuo-ku, Fukuoka 810- \\ 0022, Japan
}

\section{A R T I C L E I N F O}

\section{Keywords:}

Microglia

Allergic airway inflammation

Early postnatal period

Synaptic pruning

Dystrophic change

Transcriptome analysis

Glucocorticoid signal

Autism spectrum disorders

\begin{abstract}
A B S T R A C T
Microglia play key roles in synaptic pruning, which primarily occurs from the postnatal period to adolescence. Synaptic pruning is essential for normal brain development and its impairment is implicated in neuropsychiatric developmental diseases such as autism spectrum disorders (ASD). Recent epidemiological surveys reported a strong link between ASD and atopic/allergic diseases. However, few studies have experimentally investigated the relationship between allergy and ASD-like manifestations, particularly in the early postnatal period, when allergic disorders occur frequently. Therefore, we aimed to characterize how allergic inflammation in the early postnatal period influences microglia and behavior using mouse models of short- and long-term airway allergy. Male mice were immunized by an intraperitoneal injection of aluminum hydroxide and ovalbumin (OVA) or phosphate-buffered saline (control) on postnatal days (P) 3, 7, and 11, followed by intranasal challenge with OVA or phosphate-buffered saline solution twice a week until P30 or P70. In the hippocampus, Iba-1-positive areas, the size of Iba-1-positive microglial cell bodies, and the ramification index of microglia by Sholl analysis were significantly smaller in the OVA group than in the control group on P30 and P70, although Iba-1positive microglia numbers did not differ significantly between the two groups. In Iba-1-positive cells, postsynaptic density protein 95 (PSD95)-occupied areas and CD68-occupied areas were significantly decreased on P30 and P70, respectively, in the OVA group compared with the control group. Immunoblotting using hippocampal tissues demonstrated that amounts of PSD95, $\alpha$-amino-3-hydroxy-5-methyl-4-isoxazolepropionic acid (AMPA) receptor 2, and N-methyl-D-aspartate (NMDA) receptor $2 \mathrm{~B}$ were significantly increased in the OVA group compared with the control group on P70, and a similar increasing trend for PSD95 was observed on P30. Neurogenesis was not significantly different between the two groups on P30 or P70 by doublecortin immunohistochemistry. The social preference index was significantly lower in the three chamber test and the number of buried marbles was significantly higher in the OVA group than in the control group on P70 but not on P30, whereas locomotion and anxiety were not different between the two groups. Compared with the control group, serum basal corticosterone levels were significantly elevated and hippocampal glucocorticoid receptor (GR) amounts and nuclear GR translocation in microglia, but not in neurons or astrocytes, were significantly decreased in the OVA group on P70 but not on P30. Gene set enrichment analysis of isolated microglia revealed that genes related to immune responses including Toll-like receptor signaling and chemokine signaling pathways, senescence, and glucocorticoid signaling were significantly upregulated in the OVA group compared with the control group on P30 and P70. These findings suggest that early postnatal allergic airway inflammation induces
\end{abstract}

\footnotetext{
* Corresponding author at: Department of Neurology, Neurological Institute, Graduate School of Medical Sciences, Kyushu University, 3-1-1 Maidashi, Higashi-ku, Fukuoka 812-8582, Japan.

E-mail address: kira@neuro.med.kyushu-u.ac.jp (J.-i. Kira).
} 
dystrophic microglia that exhibit defective synaptic pruning upon short- and long-term allergen exposure. Furthermore, long-term allergen exposure induced excitatory postsynaptic surplus and ASD-like behavior. Hypothalamo-pituitary-adrenal axis activation and the compensatory downregulation of microglial GR during long-term allergic airway inflammation may also facilitate these changes.

\section{Introduction}

Microglia, resident macrophages in the central nervous system (CNS), arise early during development from progenitors in the embryonic yolk sac and persist into adulthood. Under basal conditions, microglia display ramified morphology and dynamically sense and respond to the environment to maintain neuronal homeostasis. In addition to their immunomodulatory role, microglia regulate synapses by facilitating synaptogenesis or eliminating excessive synapses, termed "synaptic pruning", which primarily occurs from the postnatal period to adolescence (Petanjek et al., 2011; Reemst et al., 2016; Semple et al., 2013). Previous studies demonstrated that synaptic pruning is essential for normal brain development (Paolicelli et al., 2011). Indeed, the disruption of this process culminates in synaptic overgrowth and behavioral deficits that mimic autism spectrum disorder (ASD) (Zhan et al., 2014).

Recent studies have focused on the inflammatory aspects of microglia because of their principal roles in neuroinflammation related to neuropsychiatric disorders. Epidemiological studies reported a strong correlation between the risk of ASD and either maternal or infantile allergic/atopic disorders including asthma, eczema, and food allergies (Chen et al., 2014b; Patel et al., 2020; Theoharides, et al., 2016). However, whether allergic inflammation alters microglial function and contributes to the development of such neuropsychiatric conditions and the mechanisms involved is unclear. One study demonstrated that the acute induction of allergic asthma in adult mice attenuated hippocampal microglial activation (Klein et al., 2016) whereas another study reported the activation of spinal microglia in adult asthma and atopic dermatitis models (Yamasaki et al., 2016). Regarding the developing brain, prenatal maternal allergic immune-activation in rats reduced microglial activation and decreased male-typical copulatory behavior in male offspring during adulthood (Lenz et al., 2019), which suggests that allergy has modulatory effects on microglia during development when they are more sensitive to environmental stimuli, which allows them to respond better to potential injury (Paolicelli and Gross, 2012).

Because the postnatal period is critical for synaptic maturation by microglia, we hypothesized that allergic inflammation during the early postnatal period alters microglial functions, thereby causing aberrant behavior later in life. In this study, given that timing or duration are important for the pathogenic stimulation of microglia (Bilbo et al., 2008; Paolicelli and Gross, 2012), we investigated whether early postnatal allergic inflammation affected microglial functions related to normal brain development, particularly synaptic pruning, using a two-time point ovalbumin (OVA) allergic asthma mouse model, and characterized how early postnatal allergy modulates microglial function and mouse behavior.

\section{Methods}

\subsection{Animals}

Wild type C57BL/6J female and male mice (10-12 weeks of age) were obtained from Japan SLC (Hamamatsu, Japan). After mating, female mice were checked for pregnancy and housed individually if confirmed. The day of birth was defined as postnatal day 0 (P0) and only male offspring were selected for allergy induction and subjected to further analyses. Offspring of both sexes and their mother were housed together until weaning on P21. Male mice were separated after weaning and housed in $23-\times 14-\times 14-\mathrm{cm}$ cages $(3-5$ per cage). Food and water were provided ad libitum. The room was maintained at a constant temperature $\left(20-24{ }^{\circ} \mathrm{C}\right)$ on a 12 -h light/dark cycle (lights on at $8 \mathrm{am}$ ). Animal care and experimental procedures were conducted according to the institutional guidelines and approved by the Kyushu University ethics committee (reference number: A20-151-0).

\subsection{Neonatal sensitization and asthma induction}

The experiment protocols are summarized in Fig. 1. The neonatal asthma model induced by intranasal administration was performed as previously described with some modifications (Hamada et al., 1999; Saglani et al., 2009). Briefly, on P3 and P7, male pups were selected and sensitized by the intraperitoneal (i.p.) injection of $5 \mu \mathrm{g}$ chicken OVA (Grade V, Sigma-Aldrich, A5503) and $1 \mathrm{mg} \mathrm{Al}(\mathrm{OH})_{3}$ (Sigma-Aldrich, 239186). On P11, male pups were sensitized again by an i.p. injection of $10 \mu \mathrm{g}$ OVA and $1 \mathrm{mg} \mathrm{Al}(\mathrm{OH})_{3}$. From the following day, male pups were intranasally challenged with $0.25 \%$ OVA in phosphate-buffered saline (PBS) twice a week. The total volume of OVA solution for intranasal administration was adjusted to 10,20 , or $40 \mu \mathrm{l}$ on P12-20, P21-P27, and $\mathrm{P} 28-70$, respectively. Control animals were injected with $1 \mathrm{mg} \mathrm{Al}(\mathrm{OH})_{3}$ only and intranasally challenged with PBS in the same manner as the experimental group. To minimize handling and physical stress, mice were briefly anesthetized with isoflurane when challenged. Pups were immediately returned to their nursing mother after injection and challenged before weaning. All procedures were performed within 1-2 min. To avoid the downregulation of airway inflammatory responses during chronic challenge and to reinforce airway inflammation (Germundson et al., 2018; Guo et al., 2013; Kumar et al., 2008), mice were intranasally administered 2.5\% OVA in PBS at 6 and 9 weeks of age. Mice were euthanized for sample collection on P30 and P70, the day after their last OVA exposure.

\subsection{Lung pathology}

Mice were sacrificed 1 day after the last intranasal OVA exposure on P30 and P70. The left lung was isolated and immersed in 4\% paraformaldehyde (PFA) overnight, then sliced sagittally at $5-\mu \mathrm{m}$ thickness and embedded in paraffin. Sections were stained with hematoxylin and eosin (HE) and periodic acid-Schiff (PAS). Goblet cell hyperplasia was quantified as a mucus score as previously described with a slight modification (Myou et al., 2003). Briefly, four to six bronchioles with internal diameters of $150-300 \mu \mathrm{m}$ in each section were randomly selected and the percentage of PAS-positive mucus-containing cells in the epithelial cells was determined as follows: grade 0 (no goblet cells); grade $1,<25 \%$; grade $2,25 \%-50 \%$; grade $3,50 \%-75 \%$; and grade 4 , greater than $75 \%$ staining. We counted peribronchial and perivascular leukocyte infiltrates on the $\mathrm{HE}$ sections as inflammatory cells and determined inflammatory scores as follows (Lu et al., 2019): grade 0, no inflammatory cells; grade 1, occasional cuffing with inflammatory cells; grade 2, most bronchi or vessels surrounded by a thin layer (1-5) of inflammatory cells; and grade 3 , most bronchi or vessels surrounded by a thick layer (more than five cells).

\subsection{Bronchoalveolar lavage collection}

We examined the infiltration of inflammatory cells in bronchoalveolar lavage fluid (BALF) to confirm the presence of airway inflammation. On P30 and P70, animals were sacrificed and their lungs were gently lavaged with $0.5 \mathrm{~mL}$ of $0.9 \%$ saline twice via a tracheal 
cannula. BALF was cytocentrifuged at $800 \mathrm{rpm}$ at $4^{\circ} \mathrm{C}$ for $10 \mathrm{~min}$ and the smear was stained with Diff-Quick solution. Differential BALF cell counts were performed with a microscope at $\times 400$ magnification and presented as a percentage for each cell type.

\subsection{Three chamber test (TCT)}

All behavioral tests were conducted the day after the last intranasal OVA exposure at 3 and 9 weeks of age to avoid acute stress due to handling. All examinations were conducted between 11 am and $7 \mathrm{pm}$. Social interaction was examined by TCT (Lo et al., 2016; Malkova et al., 2012). The apparatus comprised a box consisting of three empty chambers $(20 \times 40 \mathrm{~cm})$ with clear Plexiglas walls. Access was regulated by an opening between chambers that could be closed with dividing walls. First, the outer chambers were closed from the middle chamber by the dividing walls. A subject mouse was then placed into the middle chamber and the walls were removed. The mouse was allowed to roam freely in all three chambers for a 10-min habituation period before the Plexiglas walls dividing the chamber were reinstalled. Then, the mouse was put back into the middle chamber. After that, a wire cup-container was placed in the middle of each of the two outside chambers. One cup, containing a stranger stimulus mouse, was placed in the "social chamber" and another empty cup was placed in the chamber on the other side in the "non-social chamber". Both dividing walls were then removed simultaneously. Automated video tracking software (Smart@ v.3.0.05, Panlab Harvard Apparatus $\left.{ }^{\circledR}\right)$ recorded the time spent in each chamber for $10 \mathrm{~min}$ for every mouse. Habituation sessions were additionally recorded to investigate potential side preferences. Between sessions, the chambers and wired containers were thoroughly cleaned with $70 \%$ ethanol. Social preference was quantified and presented as follows: [(total time in social chamber/(total time in social chamber + non-social chamber) $\times 100 \%)]($ Lo et al., 2016).

\subsection{Marble burying test (MBT)}

To investigate repetitive and perseverative behavior, MBT was conducted as described by Malkova et al., 2012. This test makes use of the natural tendency of mice to perform burying behavior (Angoa-Pérez et al., 2013) and it has been established that excessive behavior in the form of repetition or perseverance can be assessed by the MBT. Mice were habituated for $10 \mathrm{~min}$ in a $31-\times 20-\times 16-\mathrm{cm}$ cage containing 4 cm-deep chipped wood bedding. After habituation, mice were returned to their cage and 20 black glass marbles $(1.5 \mathrm{~cm}$ diameter) were placed on the bedding, spread evenly apart from each other in a $4 \times 5$ pattern. The mice were placed back into the cage for $10 \mathrm{~min}$ and the number of marbles buried by more than $50 \%$ of bedding was counted.

\subsection{Open field test (OFT)}

The OFT was performed to examine the effects of allergen exposure on locomotion and anxiety of mice. The open field had a dimension of $50 \times 50 \mathrm{~cm}$ and was surrounded by $29-\mathrm{cm}$-high walls. Mice were placed
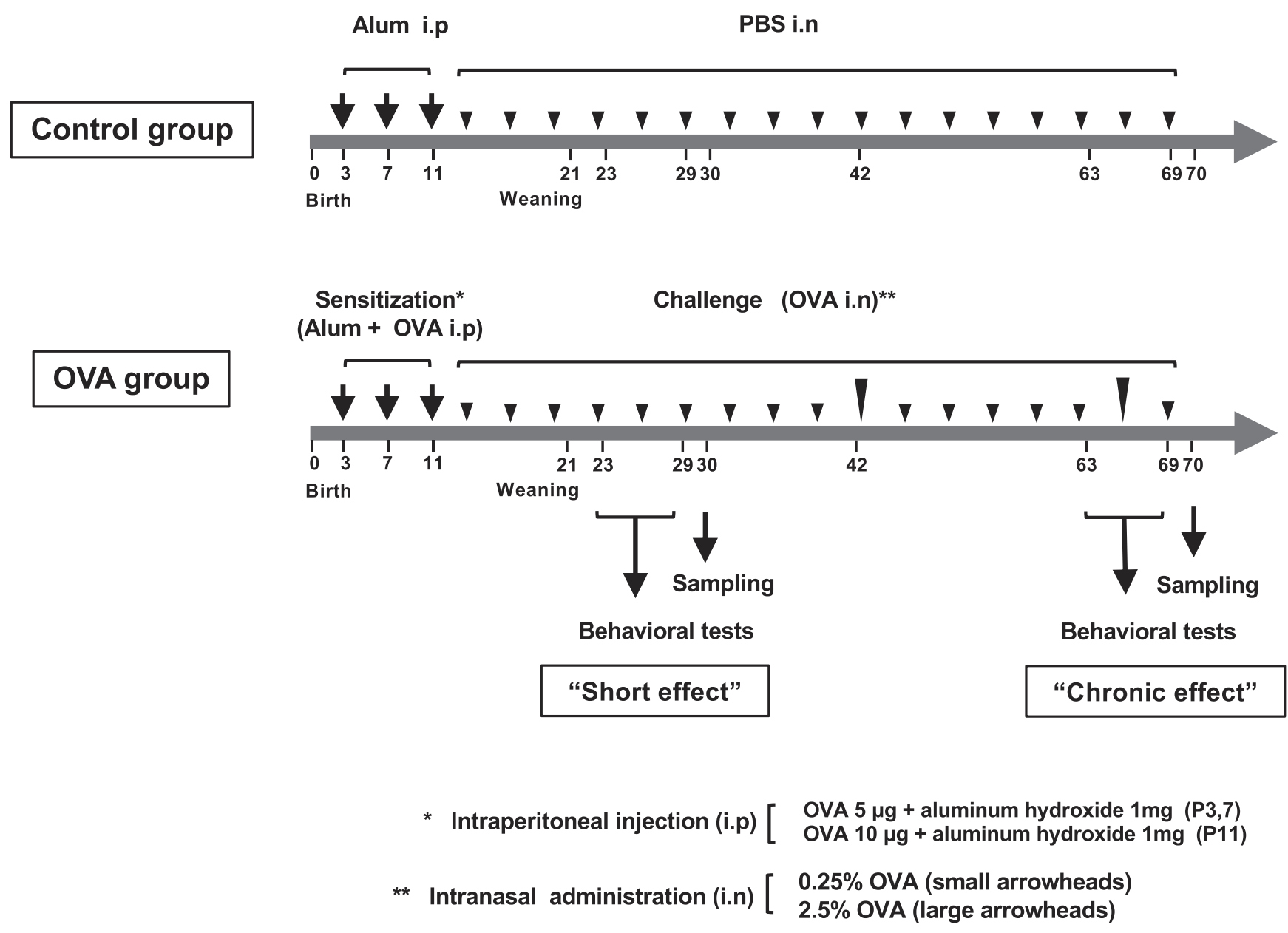

Fig. 1. Experimental protocols to assess the short- and long-term effects of allergic airway inflammation on microglia from the early postnatal period to puberty and adolescence, respectively. The black arrows indicate an intraperitoneal injection of aluminum hydroxide (1 mg on P3, P7, and P11) and ovalbumin (OVA) (5 $\mu \mathrm{g}$ on P3 and P7, and $10 \mu \mathrm{g}$ on P11) in neonates. The small arrowheads represent intranasal $0.25 \%$ OVA or PBS administration twice a week. The large arrowheads indicate intranasal 2.5\% OVA administration at 6 and 9 weeks of age to avoid the downregulation of airway inflammatory responses during long-term challenge. 
in the center of an empty field and allowed to move freely for $10 \mathrm{~min}$. The automated software divided the field into three virtual zones: outer, middle, and inner zones, and recorded the time spent in each of the three zones. Because rodents typically prefer the outer area of an open field, mice with increased anxiety show decreased locomotion and a preference to stay close to the peripheral zone (Prut and Belzung, 2003; Xie et al., 2018). We quantified the time spent in the center zone, a 16.8- $\times$ $16.8-\mathrm{cm}$ square in the center of the open field arena. Additionally, locomotion was measured by assessing the hypo- or hyperactivity of mice by recording the total distance traveled in $10 \mathrm{~min}$ using the same automated software. The field was cleaned with $70 \%$ ethanol between sessions.

\subsection{Enzyme-linked immunosorbent assay (ELISA)}

Blood was collected on P30 and P70 and centrifuged at 3,000 $\times g$ at $4{ }^{\circ} \mathrm{C}$ for $10 \mathrm{~min}$. Serum samples were stored at $-80^{\circ} \mathrm{C}$ until use. A mouse anti-OVA-IgE ELISA kit (AKRIE030, Shibayagi, Gunma, Japan) was used for the quantification of OVA-IgE levels in mouse serum. Basal serum corticosterone was also measured using a corticosterone ELISA kit (ADI900-097, Enzo, Life sciences) according to the manufacturer's protocol. Optical density for each well was measured at $450 \mathrm{~nm}$ for serum OVAIgE and at $405 \mathrm{~nm}$ for basal serum corticosterone by a luminometer (MTP-800AFC, Corona Electric, Ibaragi, Japan; and iMark, BioRad, Hercules, CA, respectively). Blood sampling of serum corticosterone was conducted between 11 am and 3 pm to minimize the effects of circadian rhythm (Gong et al., 2015).

\subsection{Multiplexed fluorescent bead-based immunoassay}

For cytokine measurements, supernatants of BALF and plasma were collected from OVA and control group mice on P30 and P70. Briefly, samples were centrifuged as follows: BALF for $10 \mathrm{~min}$ at $1,000 \mathrm{rpm}$ at $4{ }^{\circ} \mathrm{C}$ and blood for $10 \mathrm{~min}$ at $3,000 \times g$ at $4{ }^{\circ} \mathrm{C}$, and then stored at $-80^{\circ} \mathrm{C}$ until use. All assays were performed using a Bio-Plex Pro mouse cytokine kit (Bio-Rad, Hercules, CA, USA), according to the manufacturer's instructions. All samples were run in duplicate and were assayed for IL-1 $\beta$, IL-2, IL-4, IL-5, IL-6, IL-10, IL-13, IL-17, C-C motif chemokine 11 (CCL11)/eotaxin, granulocyte macrophage colony-stimulating factor (GM-CSF), interferon (IFN)- $\gamma$, and tumor necrosis factor (TNF)- $\alpha$. Results were expressed as $\mathrm{pg} / \mathrm{ml}$.

\subsection{Brain tissue preparation}

Mice were anesthetized with an i.p. injection of pentobarbital and inhalation of isoflurane, sacrificed, and transcardially perfused with icecold PBS. The brain was removed and halved sagittally. One hemisphere was fixed with $4 \%$ PFA in $0.1 \mathrm{M}$ PBS overnight at $4{ }^{\circ} \mathrm{C}$ and paraffinembedded for immunohistochemical study. The hippocampus was dissected from another hemisphere and flash-frozen with nitrogen liquid and kept at $-80{ }^{\circ} \mathrm{C}$ until used for immunoblotting analysis. In the present study, we focused on the hippocampus because ASD affects specific brain structures including the limbic system and cerebellum, rather than the entire structure, as shown neuropathologically (Blatt, 2012; Raymond et al., 1995). Furthermore, a previous study reported that allergic airway inflammation reduced numbers of microglia and induced microglial alterations in the hippocampus (Klein et al., 2016). We also examined the cell number and percentage area of Iba-1-positive cells in the frontal cortex.

\subsection{Immunohistochemistry}

Immunofluorescence staining was performed for a microglial densitometry, morphology, and co-labeling study. Paraffin-embedded tissues were cut at $6 \mu \mathrm{m}$ thickness and sections were deparaffinized in xylene, rehydrated in $99 \%$ ethanol, and then subjected to antigen retrieval. Sections were autoclaved in $10 \mathrm{mM}$ citrate buffer for $20 \mathrm{~min}$ at $95{ }^{\circ} \mathrm{C}$ and then cooled to room temperature. Thereafter, sections were permeabilized with Tris- $\mathrm{HCl}$ containing $0.1 \%$ Triton and washed twice for $3 \mathrm{~min}$ with Tris-HCl. Sections were blocked with $3 \%$ bovine serum albumin or $5 \%$ normal goat serum for $30 \mathrm{~min}$, then incubated with primary antibodies. Detailed information on the primary antibodies is provided in Supplementary Table 1. The sections were washed, incubated with Alexa Fluor 488- or 594-conjugated secondary antibodies (1:500; Thermo Fisher, Rockford, IL), and then coverslipped with mounting medium including 4', 6-diamidino-2-phenylindole (DAPI) (H1200; Vector, Burlingame, CA, USA). Immunofluorescence images were captured using a BZ-X700 fluorescent microscope (Keyence, Osaka, Japan). For hippocampal neurogenesis analysis, we used the indirect immunoperoxidase method using an Elite ABC kit (PK-6101, Vector). Paraffin sections were deparaffinized and antigen retrieval was conducted as described above. After washing, the sections were blocked and incubated with a primary antibody (Ki67 and doublecortin) overnight at $4{ }^{\circ} \mathrm{C}$. After washing with PBS, sections were labeled with a streptavidin-biotin complex and enhanced according to the manufacturer's instructions. The sections were reacted with substrate $3,3^{\prime}$ diaminobenzidine tetrahydrochloride (SK-4105, Vector) for $5 \mathrm{~min}$ and mounted with Eukitt mounting media. Images were obtained using a BX51 microscope (Olympus, Tokyo, Japan).

\subsection{Image analysis}

Microglial cell numbers and immune-reactive areas were determined using Fiji software (National Institutes of Health, Bethesda, MD, USA). Briefly, high-resolution images $(1920 \times 1440$ pixels $)$ were randomly captured in several areas in the hippocampus (dentate gyrus and CA1-3) and frontal cortex using a $20 \times$ lens and ionized calcium-binding adapter molecule 1 (Iba-1)/DAPI co-labeled cells were manually counted and divided by the area to calculate the cell density. Similarly, immunoreactive areas in each region were thresholded, divided by the area, and represented as percentages. The mean value for each mouse was calculated. We also measured the percentages of immunoreactive areas for C-X3-C motif chemokine receptor 1 (CX3CR1) using the same method. To examine microglial morphology in the hippocampus, we performed Sholl analysis. Because of the relatively thin sections, we used the method described by Martín-de-Saavedra and Robillard with some modifications (Martín-de-Saavedra et al., 2013; Robillard et al., 2016). Briefly, Z-stack images were collected with a $60 \times$ oil immersion lens with $0.3-\mu \mathrm{m}$ step size, and only cells with a clear cell body and processes not extended beyond the edge of the field were chosen. For each animal, 10-15 microglial cells were selected from the CA1-3 hippocampal area and surrounding processes were removed manually. Each image was thresholded and analyzed with a Sholl analysis plugin in Fiji. We used the line segment tool to draw a line from the center of each soma to their longest process. We set the first shell at $6 \mu \mathrm{m}$ and subsequent shells in 1$\mu \mathrm{m}$ steps to determine the number of intersections at each Sholl radius. We also manually measured the soma size and counted the number of primary branches to calculate the Ramification index (the ratio between the maximum intersections and the number of primary branches) (Heindl et al., 2018). We quantified the microglial engulfment of synapses by double immunostaining for Iba- 1 and postsynaptic density protein 95 (PSD95), a post-synaptic marker, and lysosome expression by Iba-1/CD68 double immunofluorescence staining, respectively. We measured areas positive for PSD95 or CD68 and divided this value by the Iba-1-positive areas to represent the percentage area of microglia occupied by these markers.

We also calculated the percentages of nuclear glucocorticoid (GC) receptor (GR) positive-stained cells for each cell type (neurons stained with NeuN, astrocytes stained with $\mathrm{S} 100 \beta$, and Iba-1 stained with microglia), according to previous reports with minor modifications (Maatouk et al., 2018; 2019). Briefly, cells were characterized as those with abundant GR signals in the nucleus (nuclear GR positive-stained 
cells) and those without GR signals in the nucleus or GR signals only within the cytoplasm or process (nuclear GR negative-stained cells), which were then manually counted. The stratum radiatum lacunosum moleculare of the CA1-3 regions were selected for manual counting because cells were separable to distinct cell types by visual analysis. In each sample, 10-25 cells were manually counted for the analysis. The percentage of nuclear GR positive-stained cells was calculated by dividing the nuclear GR positive-stained cell number with the (nuclear GR positive-stained cell number + nuclear GR negative-stained cell number).

In addition, we chose cells with GR staining in the nucleus that did not overlap with other cells and then quantified the mean GR staining intensity in the nucleus as the mean of the total nuclear area with the same size as that of DAPI signals without trimming the GR positivestained areas for each cell type (neurons stained with NeuN, astrocytes stained with $\mathrm{S} 100 \beta$, and Iba-1 stained with microglia). The mean nuclear GR staining intensity values were corrected by dividing the background signal intensity as reported by Lee et al. (2016). Furthermore, we trimmed the nuclear GR positive-stained areas and calculated the percentage of nuclear GR-stained areas as the GR-positive area in the nucleus divided by the DAPI-positive area. Multiple cells per sample (5-6 cells for the mean GR staining intensity, and 4-6 cells for the percentage of nuclear GR-stained area) were measured, and the obtained values were averaged in these two analyses. We selected CA1-3 regions because of their abundant GR expression (Madalena and Lerch, 2017). We also performed double immunostaining of Iba-1 and heat shock protein 90 (HSP90), a molecular chaperone for GR into the nucleus, in the CA1-3 regions, and quantified the number of Iba- 1 and HSP90 double-positive cells. All images were recorded under the same conditions between groups and analyzed by several observers in a blind manner.

\subsection{Immunoblotting}

Immunoblotting was performed to analyze GR expression and excitatory postsynaptic markers including PSD95, $\alpha$-amino-3-hydroxy5-methyl-4-isoxazolepropionic acid (AMPA) receptor 2 (GluA2), and Nmethyl-D-aspartate (NMDA) receptor 2B (GluN2B) on P30 and P70. On P70, synaptic Ras GTPase-activating protein 1 (SynGap) and GluA1 were also examined. The hippocampus was rapidly dissected from each sample and homogenized in ice-cold radioimmunoprecipitation buffer containing a protease inhibitor cocktail, $0.5 \%$ sodium dodecyl sulphate (\#08714-04; Nacalai Tesque, Kyoto, Japan), and PhosSTOP phosphatase inhibitor cocktail (\#11836153001; Roche, Mannheim, Germany). Lysates were incubated on ice for $30 \mathrm{~min}$, sonicated, and then centrifuged at $10,000 \times g$ for $10 \mathrm{~min}$ at $4{ }^{\circ} \mathrm{C}$. The protein concentrations were determined by BCA Protein Assay Kit (Thermo Fisher Scientific K.K., Tokyo). Thereafter, samples were mixed with Laemmli buffer with heating to $95{ }^{\circ} \mathrm{C}$ for $5 \mathrm{~min}$, electrophoresed in 7.5\% mini PROTEAN precast gradient gels (\#4561026; Bio-Rad), transferred onto polyvinylidene fluoride membranes, blocked with $5 \%$ skim milk, and incubated with a primary antibody. After washing, the membranes were incubated with $0.5 \%$ horseradish peroxidase-labeled IgG for $1 \mathrm{~h}$ at room temperature. Subsequently, the membrane-bound antibodies were detected with the ECL Prime Western Blotting Detection Reagent (RPN2232; GE Healthcare, Chicago, IL) and analyzed with an image analyzer (ChemiDoc XRS System; Bio-Rad).

\subsection{Microglia cell isolation}

Microglia were isolated from PBS- and OVA-treated mice as described in our previous study (Yamasaki et al., 2016) on P30 and P70, the day after the last OVA exposure. Briefly, mice were transcardially perfused under deep anesthesia with $1 \times$ PBS and the whole brain was harvested in ice-cold $1 \times$ Hank's Balanced Saline Solution (HBSS) and homogenized by a Dounce homogenizer. Stock isotonic Percoll (10-fold dilution in $10 \times$ HBSS without $\mathrm{Ca}^{2+}$ and $\mathrm{Mg}^{2+}$ ) was added to the cell suspension to make a $30 \%$ Percoll gradient. A 70\% Percoll gradient was pipetted underneath the $30 \%$ Percoll cell solution and centrifuged at $800 \times g$ for $30 \mathrm{~min}$. Thereafter, the myelin layer was removed and the mononuclear cell interphase was isolated and resuspended in $1 \times$ HBSS. Cells were blocked with an anti-mouse CD16/32 monoclonal antibody (Sony Biotechnology, San Jose, CA) and incubated with FITC anti-mouse CD11b (Cat\#101206, BioLegend) and PerCP anti-mouse CD45 (Cat\#103130, BioLegend) for $30 \mathrm{~min}$. Cells were then sorted on an SH800 (Sony Corporation, Tokyo, Japan) by gating on $\mathrm{CD}_{11} \mathrm{~b}^{+} \mathrm{CD} 45^{\text {int }}$ as microglia. A detailed gating strategy for microglia by $\mathrm{CD} 11 \mathrm{~b}^{+} \mathrm{CD} 45^{\text {int }}$ expression is shown in Supplementary Fig. 1. The percentages of $\mathrm{CD} 11 \mathrm{~b}^{+} \mathrm{CD} 45^{\text {int }}$ cells in the brain-derived live cells did not differ significantly between the OVA and control groups on P30 and P70. Sorted cells were stored directly in RNAlater ${ }^{\mathrm{TM}}$ (Thermo Fisher Scientific, Waltham, MA, USA) and subjected to microarray analysis.

\subsection{Microarray}

Total RNA was isolated from sorted microglia using an RNeasy Mini Kit (Qiagen, Hilden, Germany) ( $n=4$ samples/group). The RNA samples were then quantified with an ND-1000 spectrophotometer (NanoDrop Technologies, Wilmington, DE) and their quality was confirmed with a 2200 TapeStation (Agilent Technologies, Santa Clara, CA). Total RNA was amplified, labeled with a GeneChip ${ }^{\circledR}$ WT Pico Kit (Thermo Fisher Scientific), and hybridized to a Clariom ${ }^{\mathrm{TM}} \mathrm{D}$ Mouse Transcriptome Array (Thermo Fisher Scientific) according to the manufacturer's instructions. All hybridized microarrays were scanned with an Affymetrix scanner. For data preprocessing, an Affymetrix Expression Console ${ }^{\mathrm{TM}}$ was used to calculate relative hybridization intensities and background hybridization values, and data normalization was performed based on the SSTTMA method. The Affymetrix probe set IDs were mapped to the corresponding gene symbol IDs and subjected to further analysis. The gene array results were uploaded to the gene expression omnibus repository (accession number: GSE1595463) on the National Center for Biotechnology Information homepage (https://www.ncbi.nlm.nih.gov/geo/).

\subsection{Gene set enrichment analysis (GSEA)}

GSEA was used to investigate the specific and functionally related changes in the gene expression of microglia because this can determine whether a priori-defined gene sets show statistically significant differences between two biological groups, despite no significant difference in the expression of specific genes contributing to the results. GSEA was performed using GSEA v.4.0.3 (https://www.gsea-msigdb.org/gsea/ index.jsp) (Subramanian et al., 2005). We directly compared all genes in the control and OVA groups for each gene set. The primary result of GSEA is the enrichment score (ES), which reflects the degree to which a gene set is overrepresented at the top or bottom of a ranked list of genes. We presented the normalized enrichment score, which is the ES value normalized by the mean ES for all permutations of the data set considering the gene set size as well as the false discovery rate and normalized $p$ value. ES was considered significant when the normalized $p$ value was $<0.05$ and the false discovery rate was $<0.25$. We used gene lists from C2 (curated gene sets) and C5 (GO gene sets) gene collections and previous papers (Ayata et al., 2018; Hickman et al., 2013).

\subsection{Statistical analysis}

All data are presented as the mean \pm SEM. The statistical significance of the difference between values was determined by one-way ANOVA with Tukey's post hoc analysis, two-way factorial ANOVA with Bonferroni's post hoc test, and the Mann-Whitney $U$ test. A $p$ value $<0.05$ was considered statistically significant. All statistical analyses were performed using Graph Pad Prism 7.0 software (Graph Pad, La Jolla, CA, USA). 


\section{Results}

\subsection{Neonatal sensitization induces allergic airway inflammation by} short- or long-term OVA exposure

First, we evaluated short- and long-term airway allergic inflammation from the early postnatal period to puberty (P30) and adolescence (P70), respectively in mouse pups. BALF analysis demonstrated eosinophils were significantly increased and macrophages were significantly decreased in the OVA group compared with the control group on P30 and P70 whereas neutrophils were significantly increased in the OVA group only on P30 compared with the control group (Fig. 2A, B). Lung pathology revealed that PAS-positive cells were abundant in bronchioles of the OVA group but not the control group (Fig. 2C). Mucus scores and inflammatory scores were significantly higher in the OVA group than in the control group on P30 and P70 (mucus score, $p<0.001$ on P30 and $p$ $<0.05$ on P70; inflammatory score, $p<0.01$ on P30 and $p<0.05$ on P70) (Fig. 2D, E). Furthermore, OVA-specific IgE in sera was significantly higher in the OVA group than in the control group $(p<0.05$ on P30 and P70) (Fig. 2F). These findings indicate that short- and long-term allergen exposure from the early postnatal period induced robust allergic airway inflammation in the OVA group but not in the control group.

\subsection{Distinct cytokine profiles after short- and long-term OVA exposure}

BALF supernatant showed a marked increase in Th2 cytokines/chemokines including IL-4 $(p<0.05)$, IL-5 $(p<0.05)$, and CCL11/eotaxin $(p=0.05)$ and proinflammatory cytokines including IL-6, TNF- $\alpha$, and GM-CSF (all: $p<0.05$ ) in the OVA group compared with the control group on P30 (Fig. 2G, Supplementary Table 2). Conversely, on P70, these changes were not observed and IL-2 and IFN- $\gamma$ were significantly lower in the OVA group than the control group $(p<0.05)$, whereas only IL-17, a Th17 cytokine, was significantly increased in the OVA group compared with the control group. Plasma cytokines in the OVA group showed similar, but milder, changes to cytokines in the BALF (Fig. $2 \mathrm{H}$, Supplementary Table 2). On P30, the OVA group showed significantly higher IL-5 $(p<0.05)$ and a tendency to have marginally higher IL-6 levels $(p=0.09)$ compared with the control group whereas only IFN- $\gamma$ was significantly higher in the OVA group compared with the control group on P70 $(p<0.05)$. These findings suggest a shift in cytokine profiles from Th2-related acute airway inflammation on P30 to Th17related chronic airway inflammation on P70 by prolonged OVA exposure, at least in the BALF, although allergic airway inflammation was histologically evident at both time points.

\subsection{Early postnatal allergic airway inflammation induces dystrophic morphological changes in microglia}

We then performed immunohistochemistry for Iba-1 in the hippocampus to examine whether short- and long-term allergic airway inflammation affected microglia. As shown in Fig. 3A-D, Iba-1-positive microglia numbers in the dentate gyrus did not differ significantly between the two groups (P30: control, $39.2 \pm 6.2$ vs OVA, $46.6 \pm 4.7, \mathrm{n}=$ $6-7$ /group, $p=0.30$; P70: control, $25.8 \pm 1.9$ vs OVA, $30.3 \pm 2.5, \mathrm{n}=$ $8-9 /$ group, $p=0.23$ ) and CA1-3 (P30: control, $93.6 \pm 4.4$ vs OVA, 72.2 $\pm 8.9, \mathrm{n}=6-7 /$ group, $p=0.09$; P70: control, $52.8 \pm 1.9$ vs OVA, $58.3 \pm$ $3.2, \mathrm{n}=8-9 /$ group, $p=0.43$ ) on P30 and P70 whereas Iba-1-positve areas in the dentate gyrus were significantly smaller in the OVA group than in the control group on P70 (P30: control, $1.81 \pm 0.39$ vs OVA, $1.92 \pm 0.28, \mathrm{n}=6$ /group, $p=0.80$; P70: control, $3.19 \pm 0.46$ vs OVA, $1.79 \pm 0.30, \mathrm{n}=7-9 /$ group, $p=0.02$ ) and in the CA1-3 on P30 and P70 (P30: control, $4.55 \pm 0.5$ vs OVA, $1.88 \pm 0.43, \mathrm{n}=6$ /group, $p<0.01$; P70: control, $3.8 \pm 0.93$ vs OVA, $1.97 \pm 0.26, \mathrm{n}=7-9$ /group, $p=0.04$ ). In addition, there was a significant decrease in Iba-1-positive areas, but not Iba-1-positive microglia numbers, in the frontal cortex on P30 and
P70 ( $p<0.05$, both) (Supplementary Fig. 2).

Morphological analysis of the hippocampus at a high magnification demonstrated the sizes of Iba-1-positive microglial cell bodies were significantly reduced in the OVA group compared with the control group on P30 and P70 ( $p<0.05$ for both) (Fig. 3E, F). Sholl analysis revealed a significant decrease in the number of intersections at all Sholl radii in the OVA group compared with the control group $(p<0.001$ on P30 and P70), which indicates a reduction in the microglial process length in the OVA group. The ramification index was also significantly lower in the OVA group than in the control group ( $p<0.01$ on P30 and $p<0.001$ on P70) (Fig. 3G, H). Notably, immunoreactive areas of CX3CR1, which is expressed by microglia and critically involved in synaptic refinement (Paolicelli et al., 2011), were also significantly decreased in the OVA group compared with the control group ( $p<0.05$ on P30 and $p<0.01$ on P70) (Supplementary Fig. 3). These observations indicated that allergic airway inflammation from the early postnatal period to puberty or adolescence reduced microglial size and ramification, although microglial numbers were unchanged.

\subsection{Short- and long-term allergic airway inflammation from the early postnatal period differentially modulates microglial functional gene expression}

To characterize alterations in microglial functional gene expression corresponding to dystrophic morphological changes on P30 and P70 by airway allergy, we performed Gene Set Enrichment Analysis (GSEA) of microglia isolated from the control and OVA groups. Genes related to immune responses in the Toll-like receptor (TLR) signaling and chemokine signaling pathways were upregulated in the OVA group compared with the control group on P30 and P70 (TLR signaling, $p<$ 0.01 on $\mathrm{P} 30$ and $p<0.05$ on P70; chemokine signaling, $p<0.05$ on P30 and $p<0.01$ on P70) (Fig. 4A, B). Notably, functional genes related to (1) engulfment, (2) sensome, (3) antigen processing and presentation, and (4) clearance showed a clear contrast between short- and long-term allergic airway inflammation. In particular, these genes were significantly downregulated on P30 but upregulated on P70 in the OVA group compared with the control group (sensome, engulfment, and clearance, $p<0.001$ on $\mathrm{P} 30$ and $\mathrm{P} 70$; antigen processing and presentation, $p<0.01$ on P30 and P70) (Fig. 4C-F).

The dystrophic morphology of OVA-microglia resembled dystrophic microglia frequently observed in aged and degenerated brains (Spittau, 2017); therefore, we used an aging-related gene set to identify gene sets significantly enriched in the OVA group compared with the control group on P30 and P70 (aging, $p<0.05$ on P30 and $p<0.001$ on P70, respectively) (Fig. 4G), which suggested that senescence-like changes were accelerated in the OVA group. A previous study demonstrated that exposure of microglia to GC led to marked shrinkage in vitro (Tanaka et al., 1997); therefore, a GC signal gene set was also analyzed to identify gene sets that were significantly enriched in the OVA group compared with the control group on P30 and P70 (response to corticosteroid, $p<$ 0.001 on P30 and $p<0.05$ on P70), which suggested that the GC pathway was activated in microglia (Fig. $4 \mathbf{H}$ ).

\subsection{Microglial engulfment and lysosomal protein expression are downmodulated by early postnatal allergic airway inflammation}

Next, microglial engulfment and lysosomal protein expression were quantified by immunostaining to confirm transcriptome changes. PSD95-occupied areas in Iba-1-positive cells were significantly decreased in the OVA group compared with the control group on P30 ( $p$ $<0.05$ ) but not P70 whereas CD68-occupied areas in Iba-1-positive cells were significantly decreased in the OVA group compared with the control group on P70 $(p<0.05)$ but not P30 (Fig. 5A, B). These observations suggest dampened synaptic engulfment on P30 and decreased lysosomal protein expression on P70 in OVA microglia. 
A
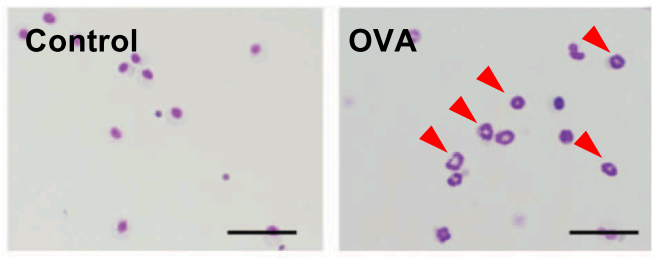

C

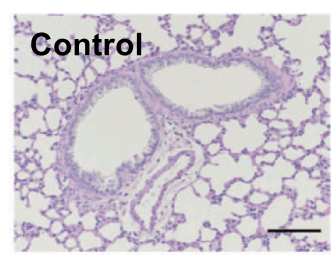

F

P30

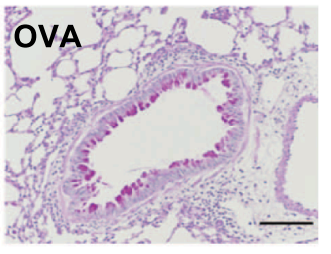

D P30

P30

B

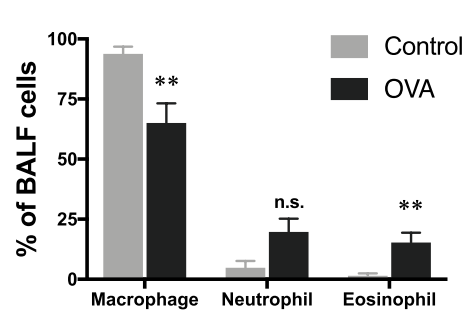

P70

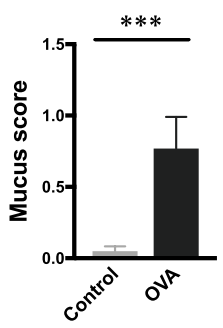

P70
P70

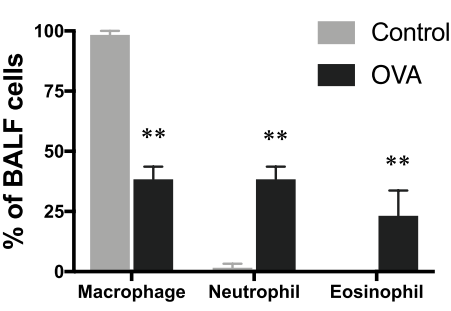

$E$

P30

P70

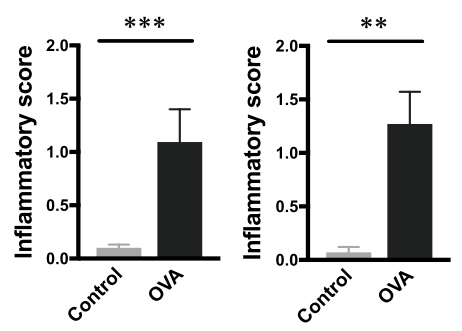

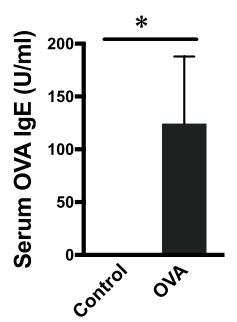

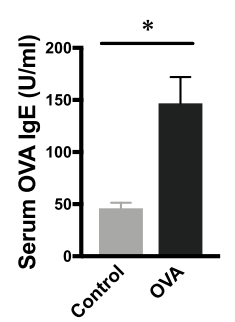

G

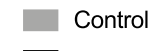

- OVA
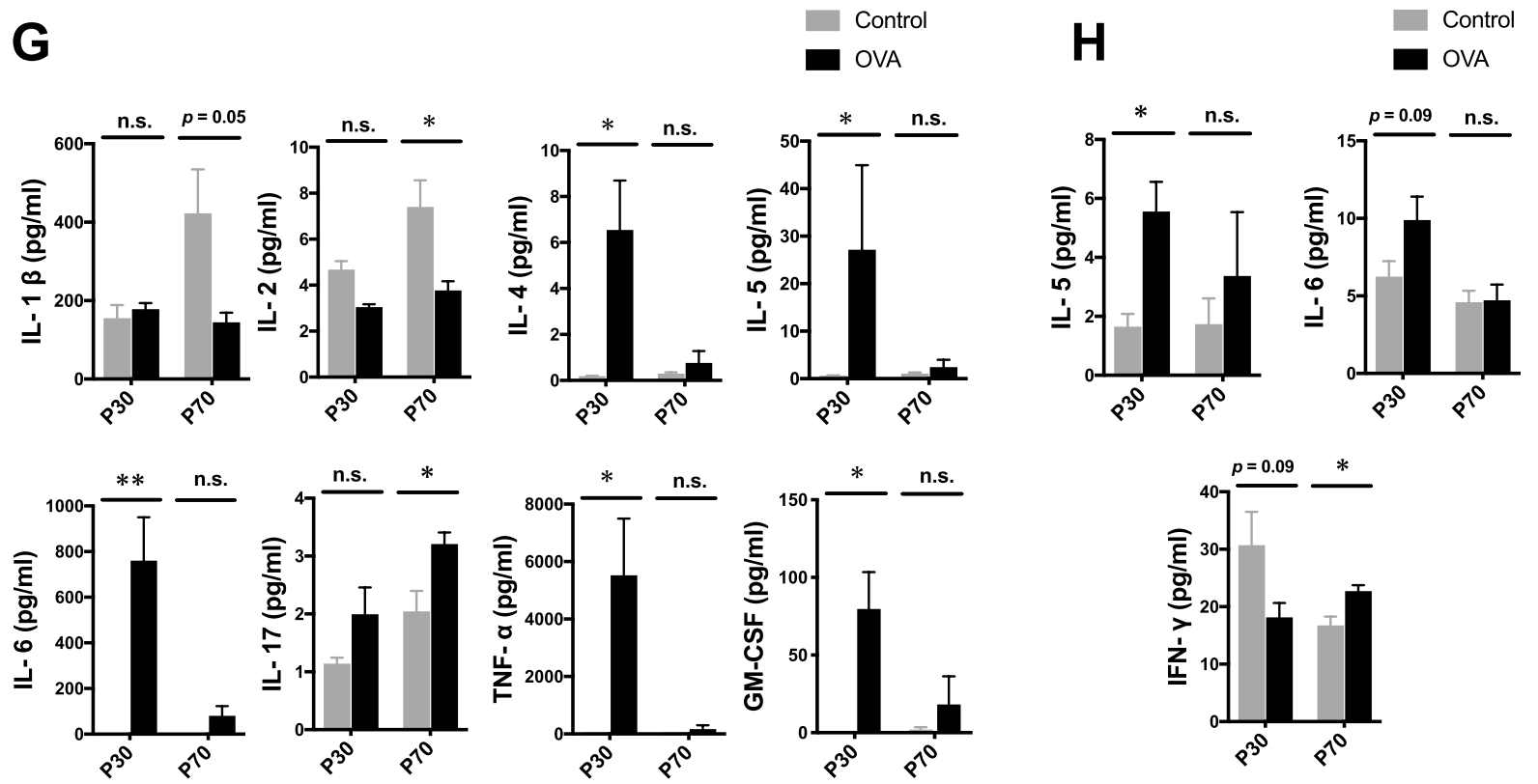

Fig. 2. Allergic airway inflammation in the short- and long-term allergen exposure models. (A) Representative cytology of bronchoalveolar lavage fluid (BALF) on P30. Red arrows depict eosinophils. Scale bars, $50 \mu \mathrm{m}$. (B) Percentages of macrophages, neutrophils, and eosinophils in BALF on P30 and P70 (P30, $\mathrm{n}=5-7$; P70, $\mathrm{n}$ $=8-9$ in each group). (C) Representative pathology of lung tissues stained by periodic acid-Schiff on P30. Scale bars, 100 $\mu \mathrm{m}$. (D) Mucus score (P30, $\mathrm{n}=7-10$; P70, $\mathrm{n}$ = 7-9 in each group) and (E) inflammatory score (P30, $\mathrm{n}=7-11 ; \mathrm{P70}, \mathrm{n}=7-9$ in each group) of lung pathology on P30 and P70, respectively. (F) Serum OVAspecific IgE (P30, $\mathrm{n}=5$; P70, $\mathrm{n}=5-6$, in each group). (G, H) Results of multiplexed fluorescent bead-based immunoassay of (G) BALF supernatant and (H) plasma from each group on P30 and P70 ( $\mathrm{n}=4$ in each group on P30 and P70). All values are shown as means $\pm \mathrm{SEM}$. n.s. $=$ not significant. ${ }^{*} p<0.05,{ }^{*} p<0.01$, and ${ }^{* * *} p<0.001$ (Mann-Whitney $U$ test). (For interpretation of the references to colour in this figure legend, the reader is referred to the web version of this article.) 

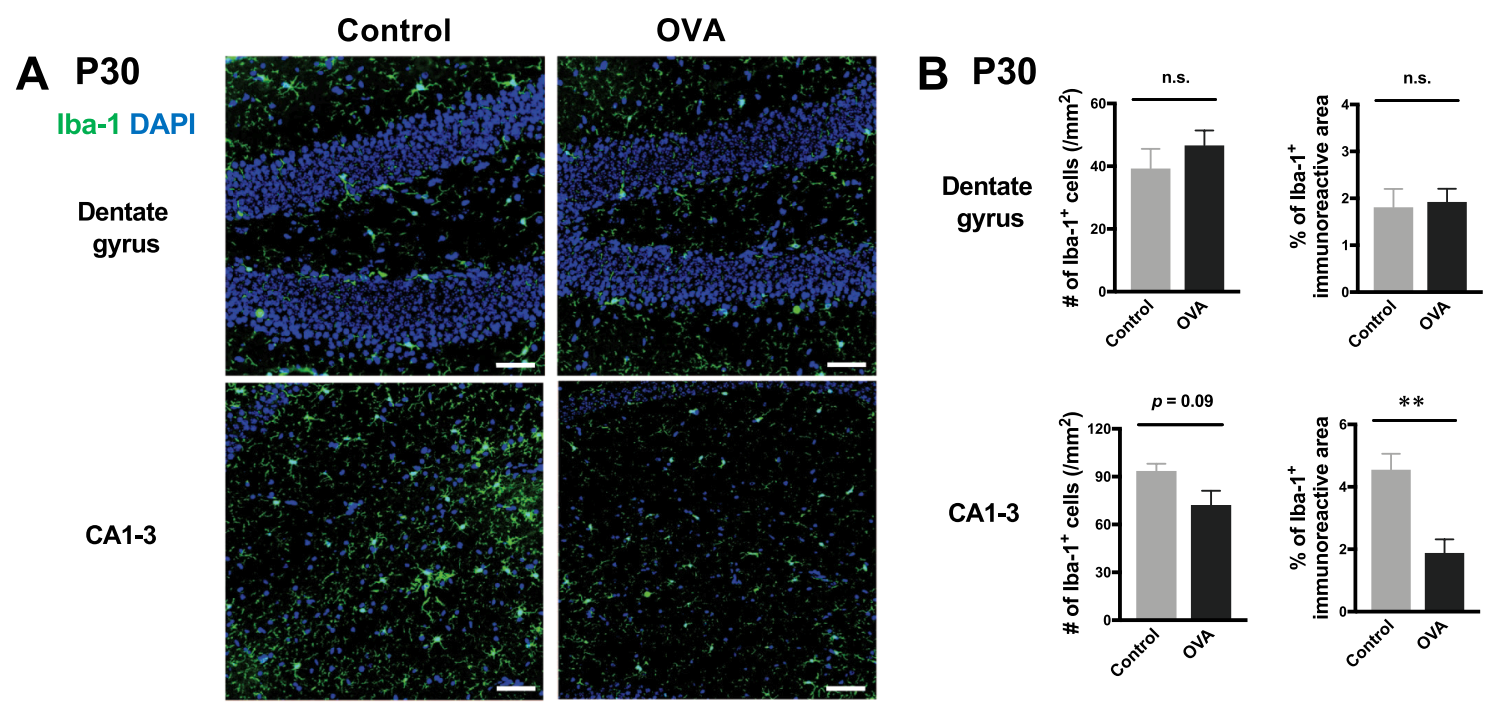

C P70

|ba-1 DAPI

Dentate

gyrus
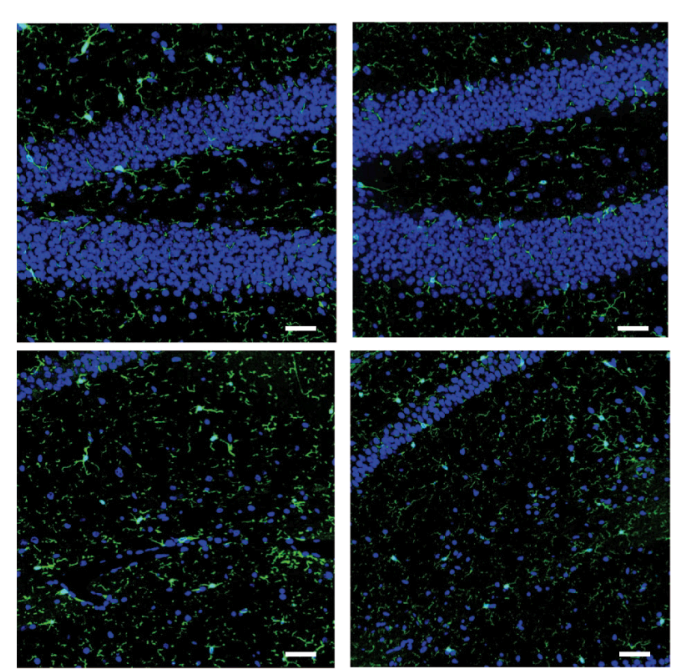

E
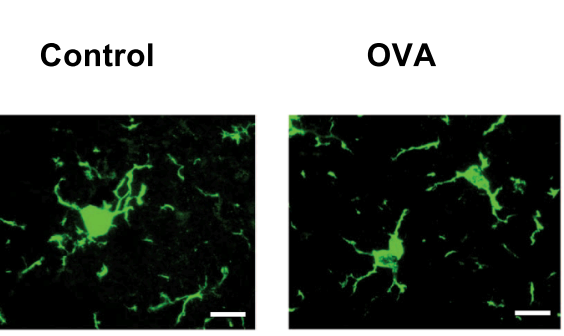

$\mathbf{F}$

P30
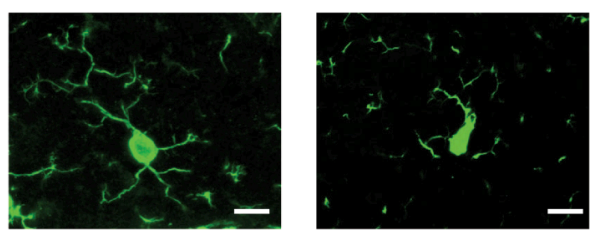
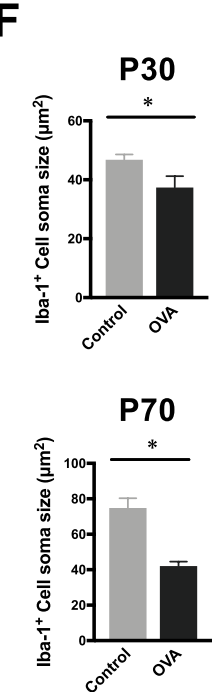

\section{P70}
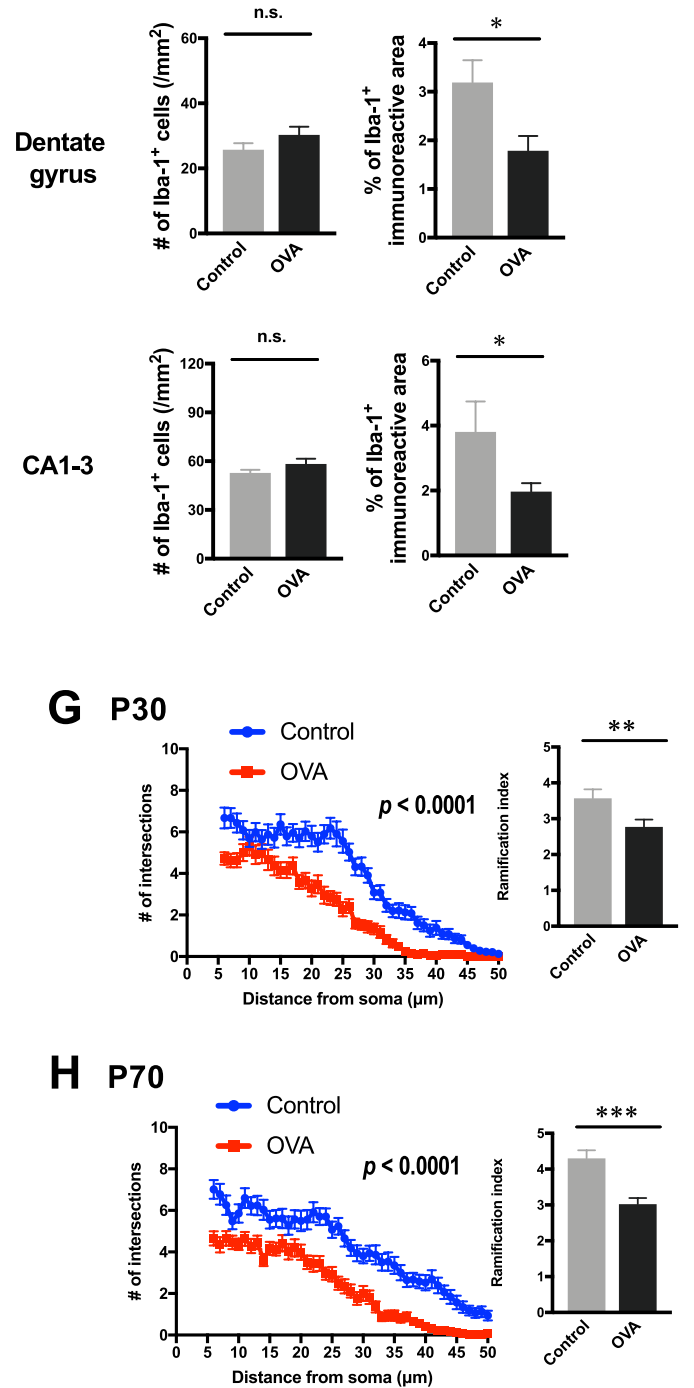

(caption on next page) 
Fig. 3. Morphological changes of microglia by early postnatal allergic airway inflammation. Representative immunofluorescence images of Iba-1-positive cells in the dentate gyrus (upper panel) and CA1-3 (lower panel) in the hippocampus on P30 (A) and P70 (C). Cell density and percentage of Iba-1-immunoreactive area in the dentate gyrus (upper graph) and CA1-3 (lower graph) on P30 (B) and P70 (D), n = 6-7/group for P30 and n = 7-9/group for P70 (2-3 images/mouse). Scale bars, $50 \mu \mathrm{m}$. (E) Representative high-magnification images of Iba-1-positive microglia in CA1-3 and (F) quantification of the microglial soma area, $\mathrm{n}=6 /$ group for P30 (4-5 images/mouse) and $n=4 /$ group for P70 (5-6 images/mouse). Scale bars, $10 \mu \mathrm{m}$. Quantification of microglial ramification complexity by Sholl analysis. An overall decrease in the number of process intersections per radius was observed in the OVA group compared with the control group on P30 (G, left graph) and P70 (H, left graph) (two-way repeated measures ANOVA, treatment $\times$ radius, main effect of treatment; $p<0.0001, \mathrm{~F}(1,60)=36.9$ on $\mathrm{P} 30$, and $p<0.0001$, $\mathrm{F}(1,66)=$ 33.9 on P70, respectively), $n=5$ /group, 10-15 images/mouse for P30, and $n=5-6 /$ group, 10-15 images/mouse for P70. The ramification index (number of max intersections/number of primary branches) was significantly reduced in the OVA group compared with the control group on P30 (G, right graph) ( $n=5 /$ group, 10-15 images/mouse) and P70 (H, right graph) $\left(\mathrm{n}=5-6 /\right.$ group, 9-15 images/mouse). All values are shown as means \pm SEM. n.s. $=$ not significant. ${ }^{*} p<0.05, * * p$ $<0.01$, and $* * * p<0.001$ (Mann-Whitney $U$ test).

\subsection{Long- but not short-term allergic airway inflammation from the early} postnatal period leads to synaptic surplus without influencing neurogenesis

Immunoblotting using hippocampal tissues showed that amounts of PSD95, GluA2, and GluN2B were significantly increased in the OVA group compared with the control group on $\mathrm{P} 70(p<0.05$ for all $)$ and that similar increasing trends were observed on P30 although these were not statistically significant (Fig. 6A, B). Amounts of another postsynaptic marker, SynGap, were also significantly increased in the OVA group compared with the control group on P70 $(p<0.05)$. Next, we examined whether neurogenesis was altered by allergic airway inflammation by the immunohistochemical analysis of doublecortin, an immature neuronal marker, and Ki67, a cell proliferation marker, in the hippocampal dentate gyrus. There were no significant changes in neurogenesis between the OVA and control groups on P30 or P70 (Fig. 6C). These findings collectively suggest that postsynaptic surplus induced by long-term allergic airway inflammation is probably attributable to the decreased engulfment and/or lysosomal expression of microglia but not enhanced neurogenesis.

\subsection{Long-but not short-term allergic airway inflammation from the early postnatal period induces autism-like behavior}

We conducted behavioral analyses to evaluate whether dystrophic microglial changes and synaptic surplus indicated by increased postsynaptic protein amounts were associated with behavioral changes in OVA mice. There was no significant difference in body weight between the OVA and control groups at the time of behavioral testing (P30: control, $15.82 \pm 0.49$ vs OVA, $14.98 \pm 0.56, \mathrm{n}=14-15$ /group; P70: control, $25.0 \pm 0.24$ vs OVA, $24.5 \pm 0.38, n=23$ /group).

The three chamber test (TCT) indicated no potential deviation between chambers in the habituation session on P30 and P70 (data not shown). On P30, the contact time in the social chamber was significantly longer than that in the non-social chamber in the OVA and control groups ( $p<0.001$ for both groups) (Fig. 7A) and there was no significant difference in the social preference index between the two groups (Fig. 7B). Conversely, on P70, the contact time in the social chamber was significantly longer than that in the non-social chamber for the control group ( $p<0.001$ ), but not for the OVA group. Consequently, the social preference index was significantly smaller in the OVA group than in the control group $(p<0.05)$ (Fig. 7A, B). The marble burying test (MBT) showed no significant difference between the two groups on P30 whereas the number of buried marbles was significantly higher in the OVA group than in the control group on P70 (Fig. 7C). The open field test (OFT) indicated no significant difference in locomotor activity (Fig. 7D) or time spent in the center of the field (indicating an anxious state) (Fig. 7E) between the two groups on P30 and P70. These findings indicate that autism-like behavioral abnormalities including decreased sociability and repetitive stereotypic behavior only appeared when allergic airway inflammation persisted from the early postnatal period to adolescence.
3.8. Long-term allergic airway inflammation from the early postnatal period alters the microglial GC pathway and activates the hypothalamopituitary-adrenal (HPA) axis

Because accumulating evidence indicates the HPA axis is involved in chronic allergic inflammation (Chen and Miller, 2007; Kalogeromitros et al., 2007) as well as ASD (Iwata et al., 2011; Lam et al., 2006), we examined the HPA axis in our model to investigate how long- but not short-term allergic airway inflammation causes ASD-like behavior.

The measurement of serum corticosterone by ELISA revealed that basal corticosterone levels were significantly higher in the OVA group than in the control group on P70 $(p<0.05)$ whereas no significant difference was found between the two groups on P30 (Fig. 8A). Next, immunoblots demonstrated that hippocampal GR amounts were significantly decreased in the OVA group compared with the control group on P70 $(p<0.05)$ but not on P30 (Fig. 8B, C).

Then, we examined nuclear GR expression in hippocampal tissues according to each cell type by the double staining of GR and antibodies to Iba-1, NeuN, or S100ß. Previous studies reported that GR is mostly localized in the nucleus compared with the cytoplasm of microglia (Maatouk et al., 2018), as well as in neurons and astrocytes in the substantia nigra (Maatouk et al., 2019). In accord with these reports, we found most nuclear GR-positive staining was in microglia, neurons, and astrocytes in the stratum radiatum lacunosum moleculare of CA1-3 (Fig. 9). The percentage of nuclear GR positive-stained microglial cells was significantly decreased in the OVA group compared with the control group on P70 $(p<0.05)$ but not on P30. No significant difference in the percentage of nuclear GR positive-stained cells was found for neurons or astrocytes between the OVA and control groups on P30 or P70. The mean nuclear GR staining intensity and percentage of nuclear GRstained area were also significantly decreased in microglia of the OVA group compared with the control group on P70 but not on P30 (Fig. 9). No significant difference in these parameters was observed in neurons or astrocytes between the OVA and control groups on P30 or P70. Double immunostaining of Iba-1 and HSP90 showed a significant increase in the number of merged cells in the OVA group compared with the control group on P70 $(p<0.05)$ whereas the number of merged cells tended to be lower in the OVA group compared with the control group on $\mathrm{P} 30$ ( $p=$ 0.06) (Supplementary Fig. 4). Collectively, these results suggest that long-term allergic airway inflammation that persisted from the early postnatal period to adolescence activated the HPA axis, and that compensatory GR downregulation occurred in hippocampal microglia. Given that HSP90 present in the cytoplasm can bind to GR (Grad and Picard, 2007), the decrease in nuclear GR despite an increase in cytoplasmic HSP90 in a fraction of microglia suggests the altered nuclear translocation of GR.

\section{Discussion}

In this study, we found that early postnatal allergic airway inflammation without maternal allergen immunization induced dystrophic microglial morphological changes upon short- and long-term allergen exposure. Furthermore, microglial functional gene expression patterns were differentially dysregulated in part according to the exposure 


\section{A Toll-like receptor signaling pathway (KEGG)}
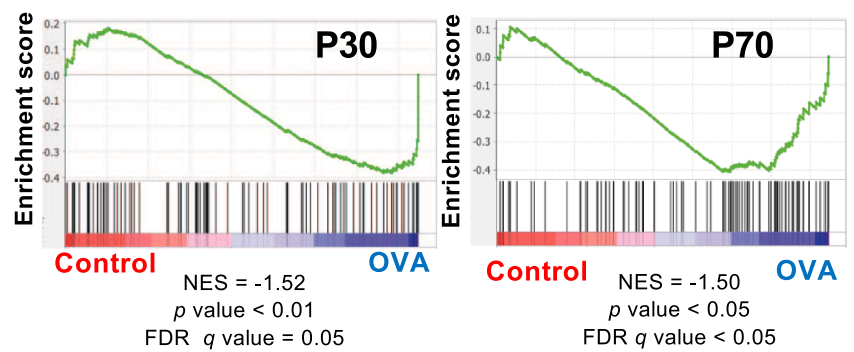

C Engulfment (Ayata et al., 2018)
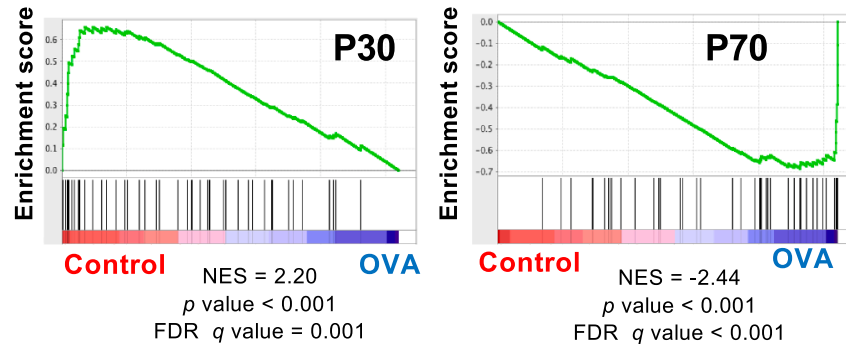

E Antigen processing and presentation (GO)
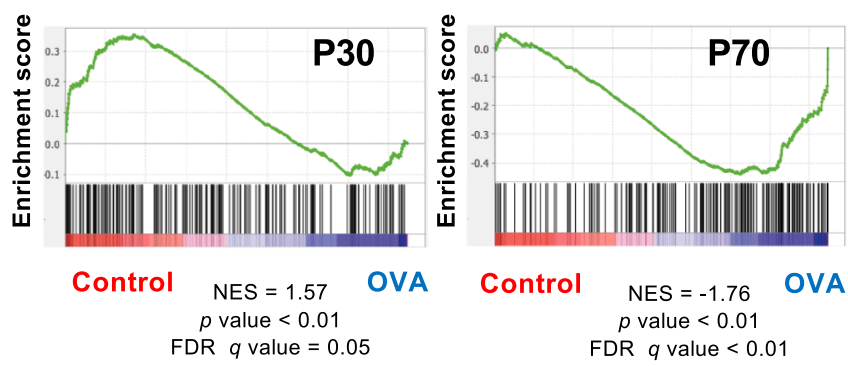

\section{G Aging (GO)}

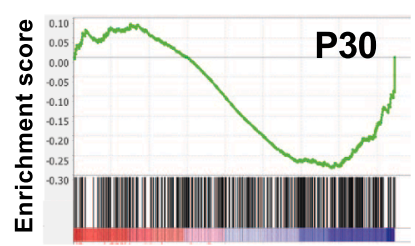

Control

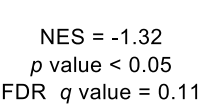

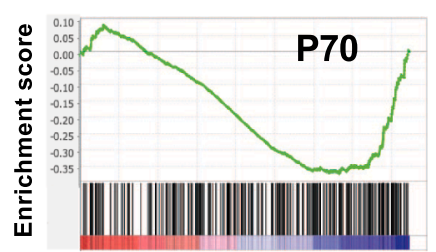

OVA Control
NES $=-1.55$
$p$ value $<0.001$

FDR $q$ value $<0.05$

B Chemokine signaling pathway (KEGG)
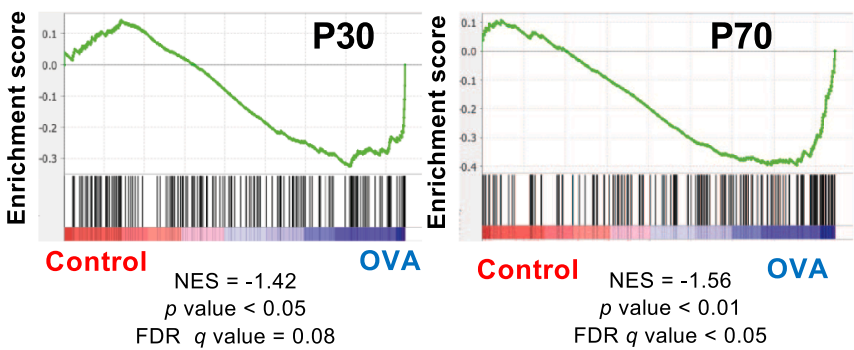

D Sensome (Hickman et al., 2013)
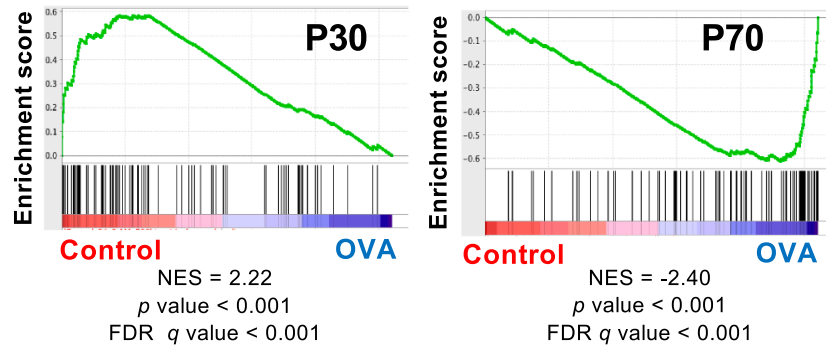

F Clearance (Ayata et al., 2018)

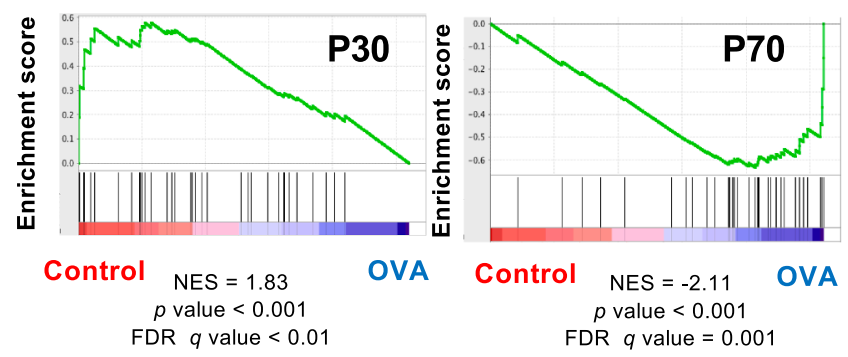

H Response To Corticosteroid (GO)

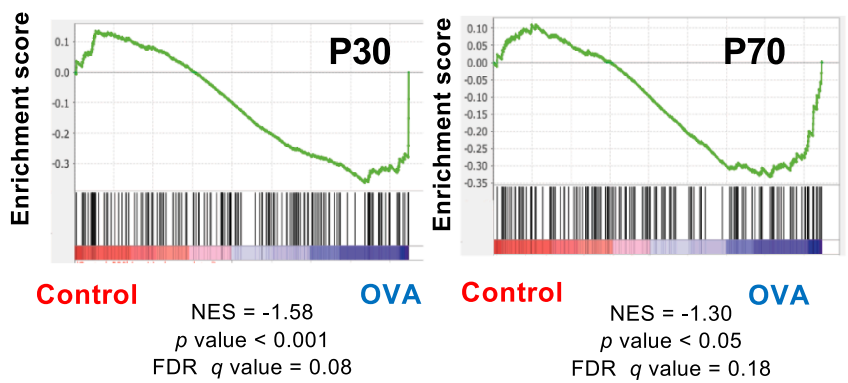

Fig. 4. Gene Set Enrichment Analysis (GSEA) of isolated microglia from whole mouse brain. Enrichment plots (green curve) show the running sum of the enrichment score (ES) for each gene set. The score at the peak of the plots is the ES for each gene set. The black bars show where the members of the gene set appear in the ranked list of genes. A predominance of black bars to the left or right side indicates that most genes are upregulated in control or OVA group mice. GSEA results revealed the upregulation of immune responses for (A) the Toll-like receptor pathway and (B) chemokine signals on P30 and P70. Short-term allergic inflammation downregulated microglial genes for (C) engulfment, (D) sensome, (E) antigen processing and presentation, and (F) clearance, while long-term allergic inflammation upregulated these functional genes. Notably, significant enrichment in genes related to (G) aging and (H) response to corticosteroid signals were observed in the OVA group compared with the control group on P30 and P70. FDR $=$ false discovery rate. NES = normalized enrichment score. Nominal $p$ values were calculated by permutation test (Subramanian et al., 2005). (For interpretation of the references to colour in this figure legend, the reader is referred to the web version of this article.) 

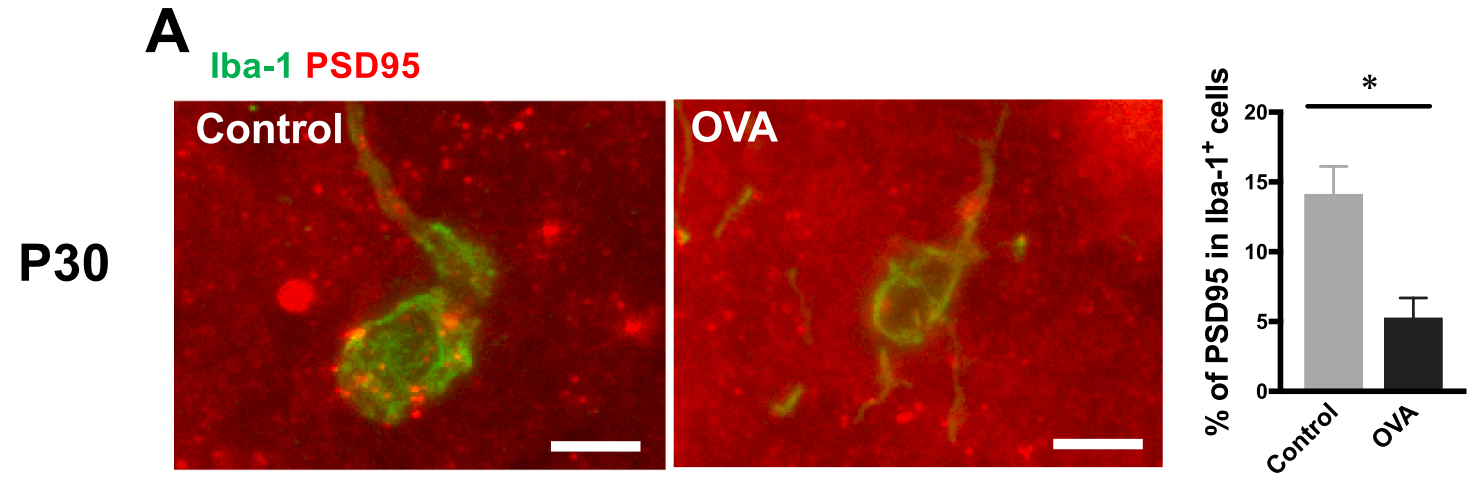

B

Iba-1 PSD95
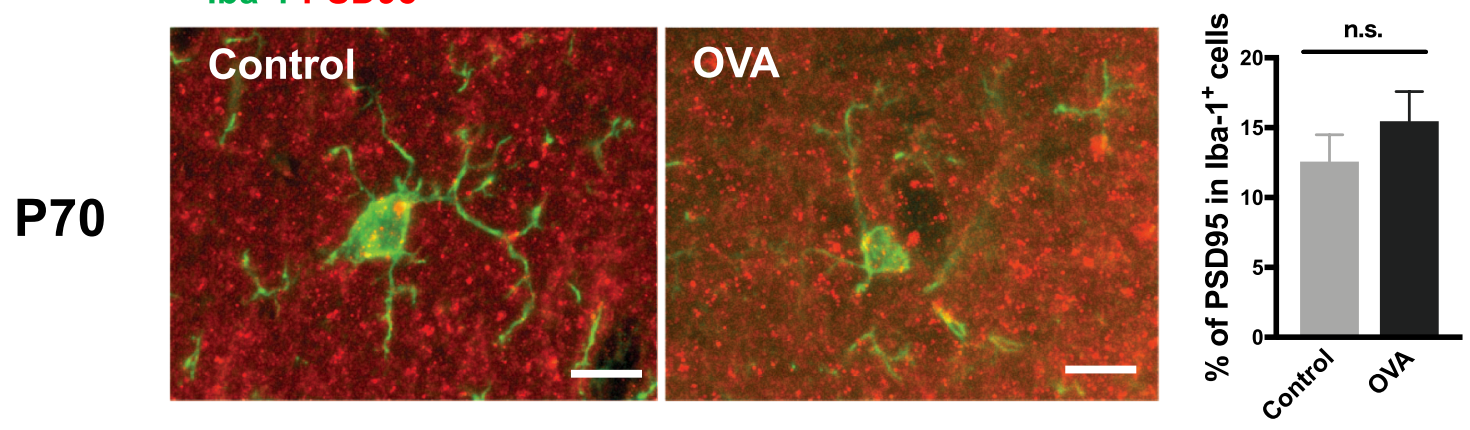

C

lba-1 CD68

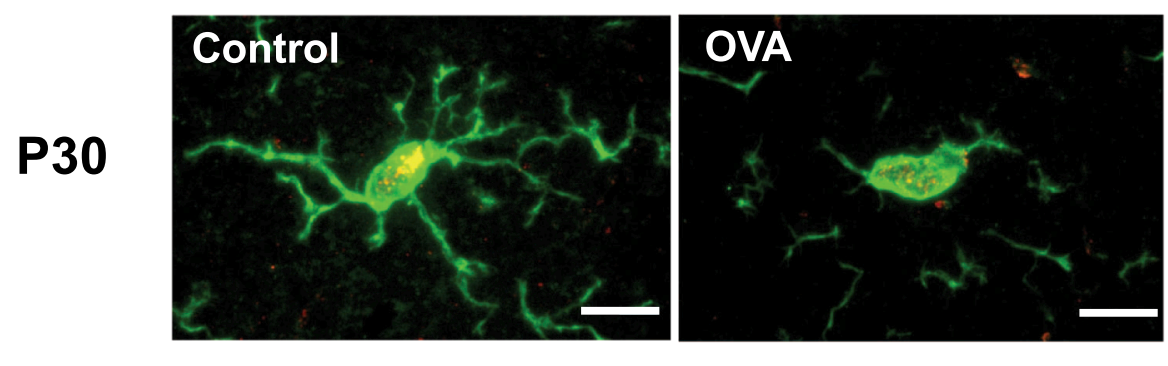

D

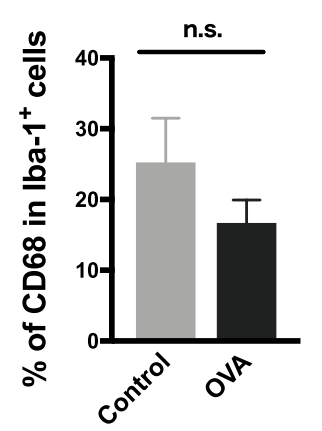

Iba-1 CD68
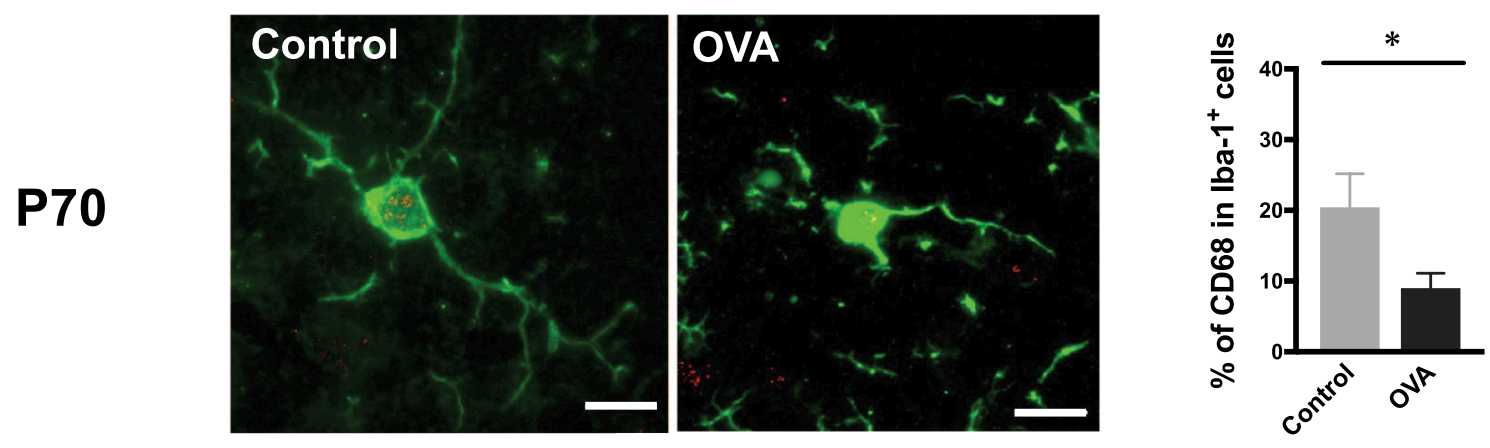

Fig. 5. Modulation of engulfment and lysosomal protein expression in hippocampal microglia by early postnatal allergic airway inflammation. Engulfment activity was analyzed by the immunofluorescence staining of hippocampal microglia co-labeled with anti-Iba-1 antibody (green) and anti-postsynaptic density protein 95 (PSD95) antibody (red) at (A) P30 and (B) P70, n = 5-6/group, 3-5 images/mouse. A significant decrease in engulfment activity was observed in the OVA group compared with the control group on P30 but not P70. Lysosome protein expression in hippocampal microglia was assessed by immunofluorescence co-staining with anti-Iba-1 antibody (green) and anti-cluster of differentiation 68 (CD68) antibody (red) at (C) P30 and (D) P70, n = 4-6/group, 3-5 images/mouse. A significant decrease in lysosome protein expression was observed in the OVA group compared with the control group on P70 but not P30. Scale bar, $10 \mu \mathrm{m}$ in all images. All values are shown as means \pm SEM. n.s. = not significant. $P$ values by Mann-Whitney $U$ test are indicated. (For interpretation of the references to colour in this figure legend, the reader is referred to the web version of this article.) 


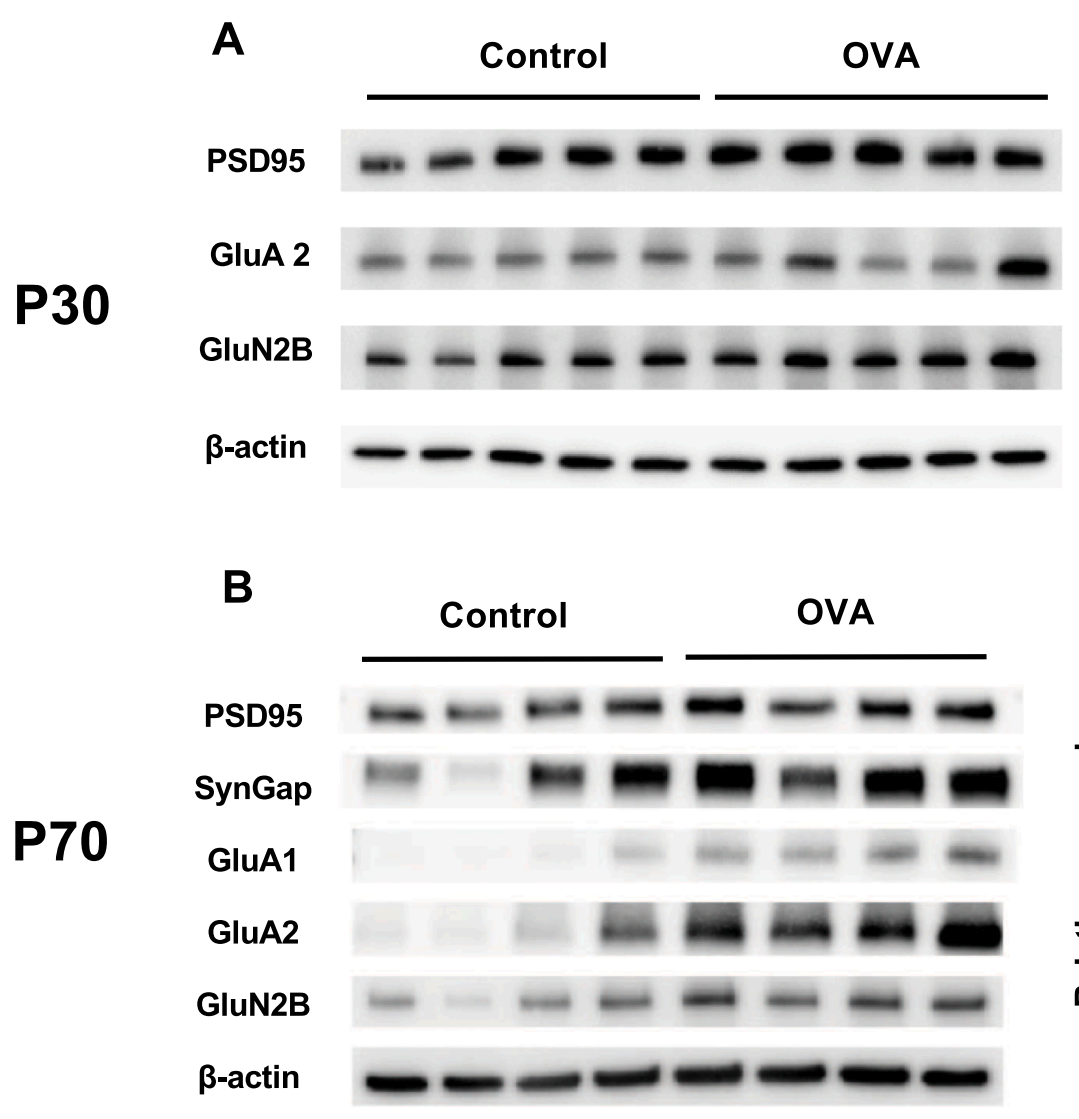

C P30
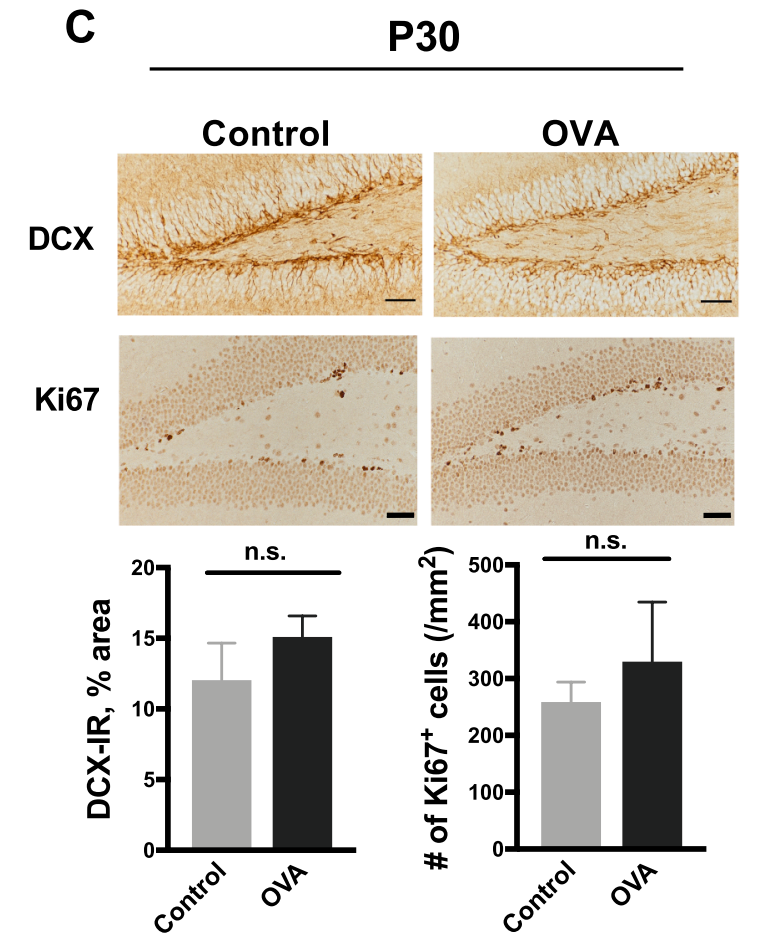
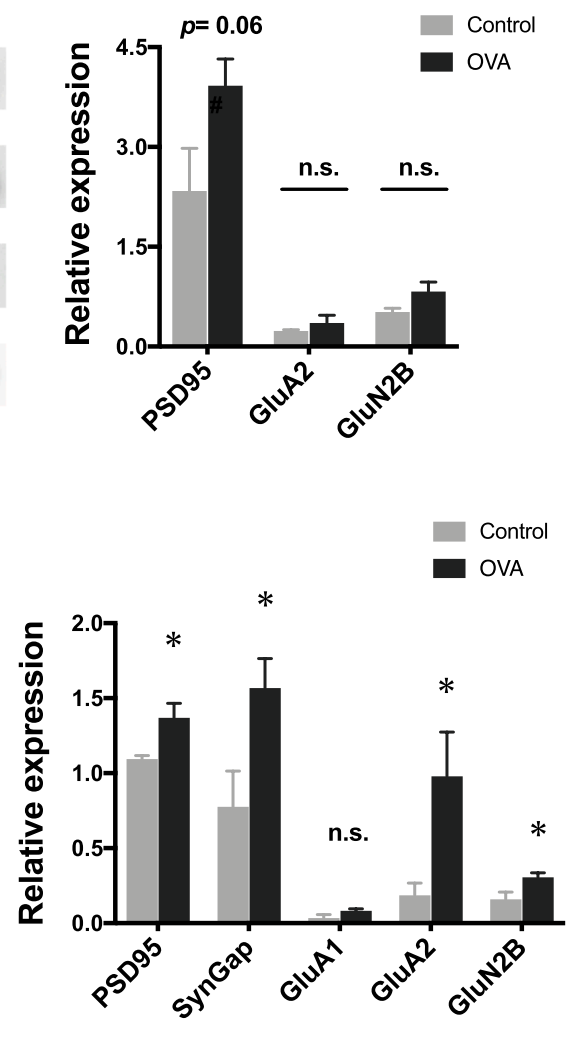

Fig. 6. Effects of short- and long-term allergic airway inflammation from the early postnatal period to puberty and adolescence, respectively on postsynaptic protein expression and neurogenesis. Immunoblot analysis of postsynaptic protein and glutamate receptors is shown at (A) P30 and (B) P70, $\mathrm{n}=4 /$ group for P30 and $\mathrm{n}=5$ / group for P70. (C) Neurogenesis was assessed by immunohistochemical analysis using anti-doublecortin (DCX) antibody ( $\mathrm{n}=5-6 /$ group for P30; $\mathrm{n}=9-10 /$ group for P70, 2-3 images/mouse) and anti-Ki67 antibody ( $n=6$ /group for P30; $n=5-6$ /group for P70, 2-3 images/mouse). Graphs show percentages of DCXimmunoreactive (IR) areas and numbers of Ki67 positive cells per $\mathrm{mm}^{2}$ in each group on P30 and P70. Scale bar, $50 \mu \mathrm{m}$ in all images. All values are shown as means \pm SEM. n.s. $=$ not significant. ${ }^{*} p<0.05$ (Mann-Whitney $U$ test). 
A

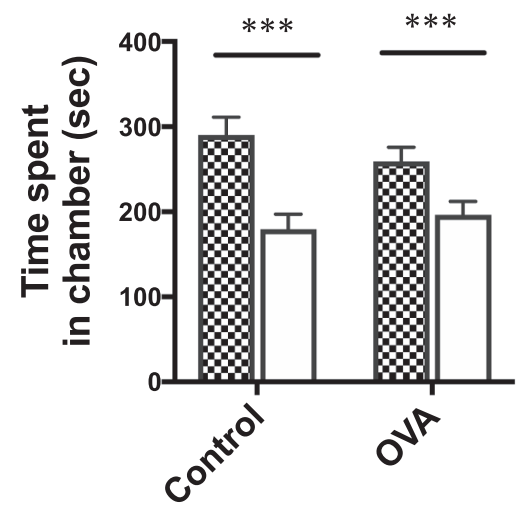

B

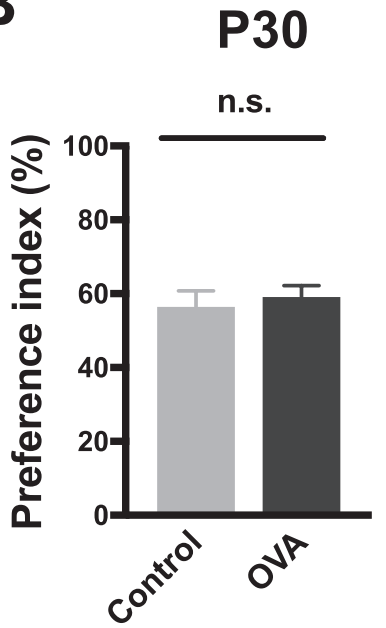

D
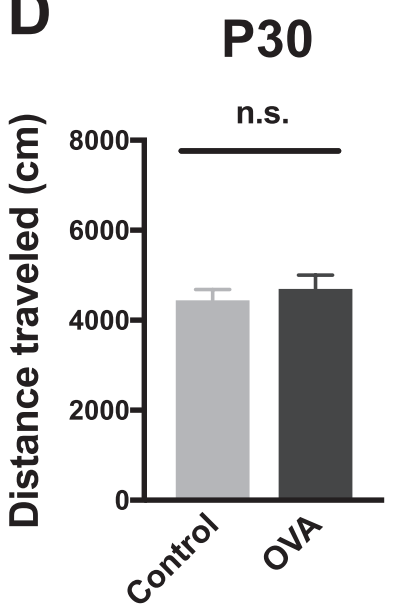

P30
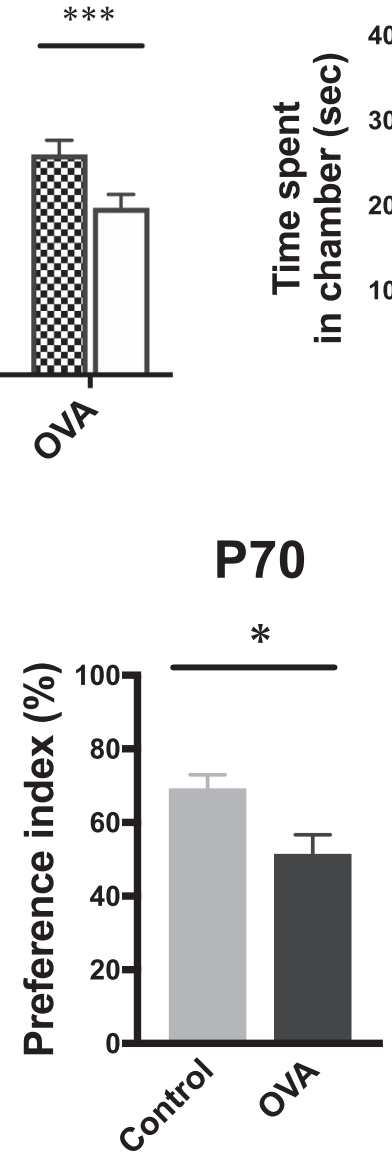

P70

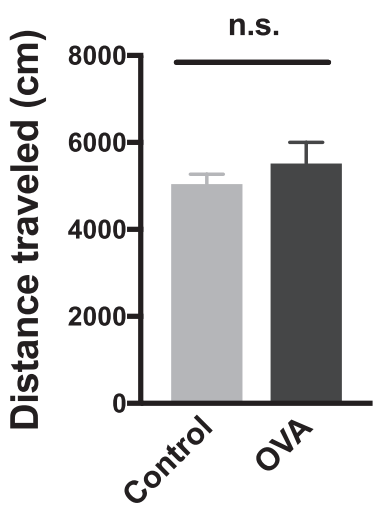

Wes Stranger

Empty

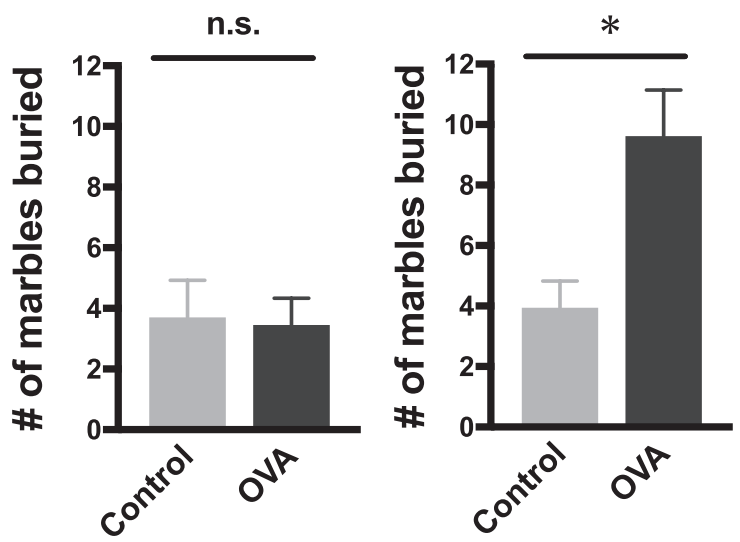

$E$

P30

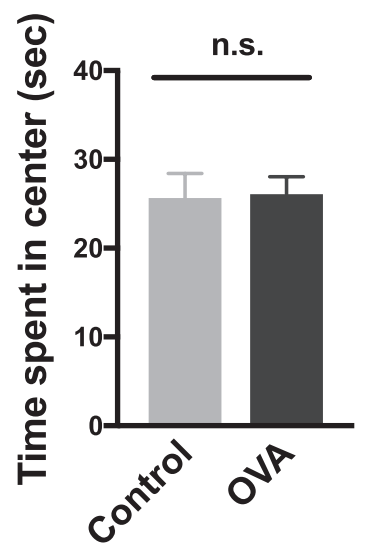

P70

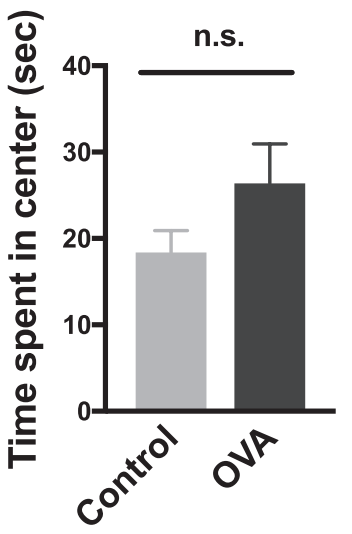

Fig. 7. Behavioral changes in mice after short- and long-term allergic airway inflammation from the early postnatal period to puberty and adolescence, respectively. (A, B) Three chamber test for sociability assessment. (A) Bar graphs show the time spent in each side chamber containing a novel mouse or empty wire cup (novel object) on P30 ( $n=21$ /group, $p<0.0001$ for main chamber effect, $p=0.686$ for main treatment effect, $p=0.175$ for chamber $\times$ treatment interaction, by two-way ANOVA) and P70 ( $n=13-15 /$ group, $p<0.0001$ for main chamber effect, $p=0.0975$ for main treatment effect, $p=0.0143$ for chamber $\times$ treatment interaction, by two-way ANOVA). ${ }^{* * *} p<0.001$ for chamber effect between stranger chamber and empty chamber by Bonferroni post-hoc test following two-way ANOVA with the factors of chamber and treatment. (B) Preference for a novel mouse is shown as the Preference index, which is calculated as [(time spent exploring stranger chamber)/(total time spent exploring stranger and empty chamber)] $\times 100 \%$ on P30 ( $\mathrm{n}=21 /$ group) and P70 ( $\mathrm{n}=13-15 /$ group), * $p<0.05$ (Mann-Whitney $U$ test). (C) Marble burying test to analyze repetitive behavior. Short-term postnatal airway allergy had no effect whereas long-term postnatal airway allergy led to increased repetitive behavior in mice, $n=17-20$ /group for P30, and $n=13-17$ /group for P70. (D, E) Open field test. Short- and long-term airway inflammation had no effect on locomotor activity (D) or anxiety-like behavior (E). All values are shown as means \pm SEM. n.s. $=$ not significant. 
A

P30

P70
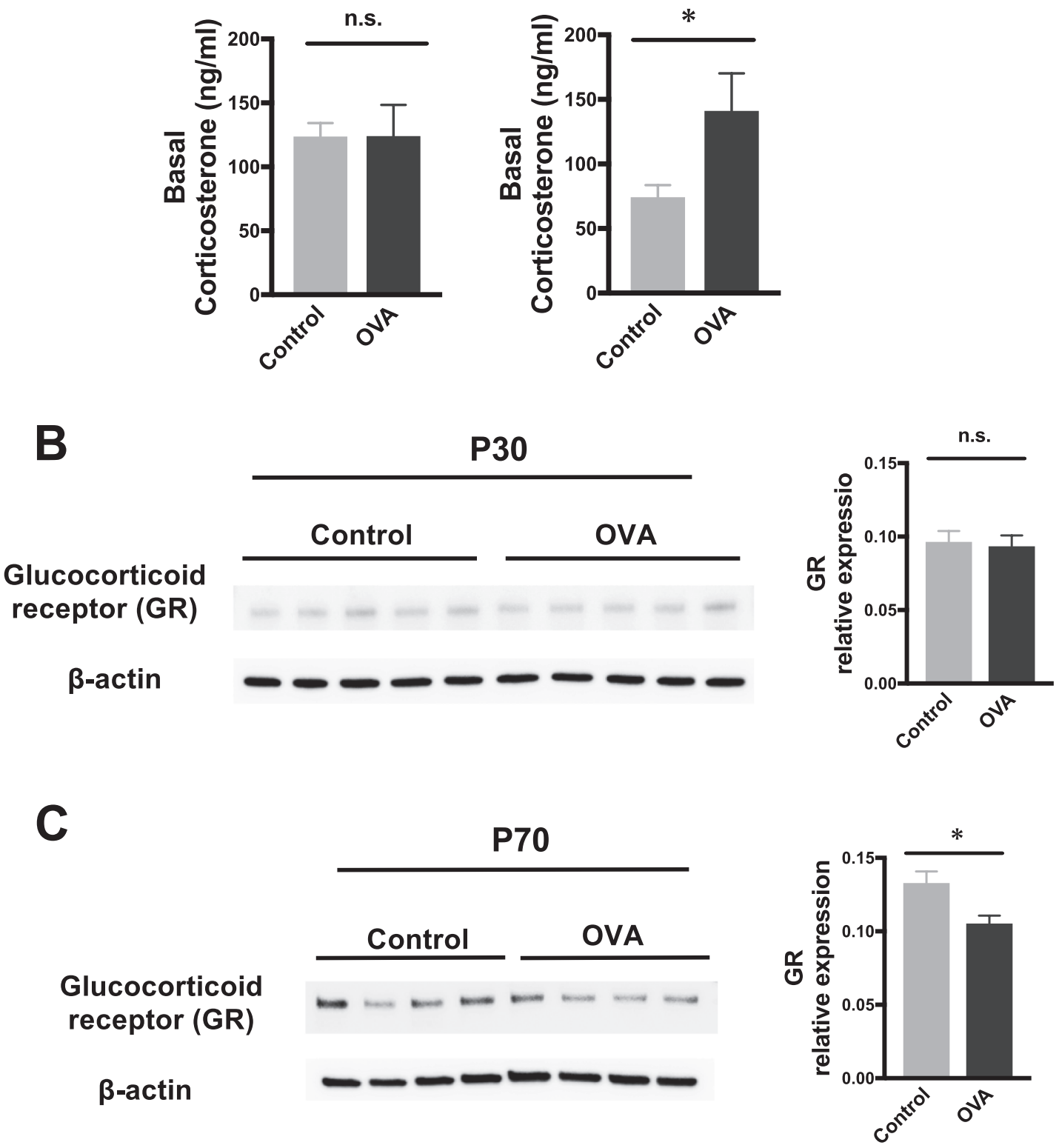

Fig. 8. Effects of short- and long-term allergic airway inflammation from the early postnatal period to puberty and adolescence, respectively on serum corticosterone levels and glucocorticoid receptor (GR) expression in hippocampal tissues. (A) Long-term allergic airway inflammation increased serum basal corticosterone levels in the OVA group compared with the control group, $n=6$ on P30 and $n=7$ on P70. Immunoblot analysis of GR in the whole hippocampus at (B) P30 and (C) P70. Downregulation of hippocampal GR is shown in OVA mice on P70 but not on P30, $n=5$ /group for P30 and $n=4 /$ group for P70. All values are shown as means \pm SEM. n.s. $=$ not significant. * $p<0.05$ (Mann-Whitney $U$ test).

period. Consequently, long- but not short-term allergic airway inflammation led to synaptic surplus without alterations in neurogenesis and manifested ASD-like behavior. HPA activation developed only after long-term allergen exposure, which might have induced compensatory GR downmodulation in microglia.

Dystrophic microglia with shrunken cell bodies and fewer ramified processes were commonly seen on P30 and P70 and were characteristic features of our model. The enrichment of immune response-related genes (i.e., Toll-like receptors and chemokine signaling), functional genes (i.e., engulfment, sensome), and senescence-related genes in sorted microglia indicated altered cellular homeostasis and the acceleration of a senescence-like process. In postmortem human ASD brains, a significant decrease of ramified microglia was reported (Lee et al., 2017).
In animal models of ASD, CX3CR1-deficient mice with deficient social interactions and increased repetitive behavior showed reduced Iba-1positive microglia with deramified morphology at 2 months of age, which was associated with defective synaptic pruning (Gyoneva et al., 2019). Interestingly, our OVA group also showed decreased CX3CR1 expression, which suggests defective microglial synaptic pruning occurs in our model (Paolicelli et al., 2011). Furthermore, microglia in an ASD marmoset model induced by the in utero administration of valproic acid had few processes and thin and segmented morphologies (Sanagi et al., 2019). Thus, we consider that early postnatal allergic airway inflammation induces dystrophic changes in microglia, which are similar to those observed in humans as well as experimental genetic and toxic ASD animal models. 


\section{A GR Iba-1 DAPI}
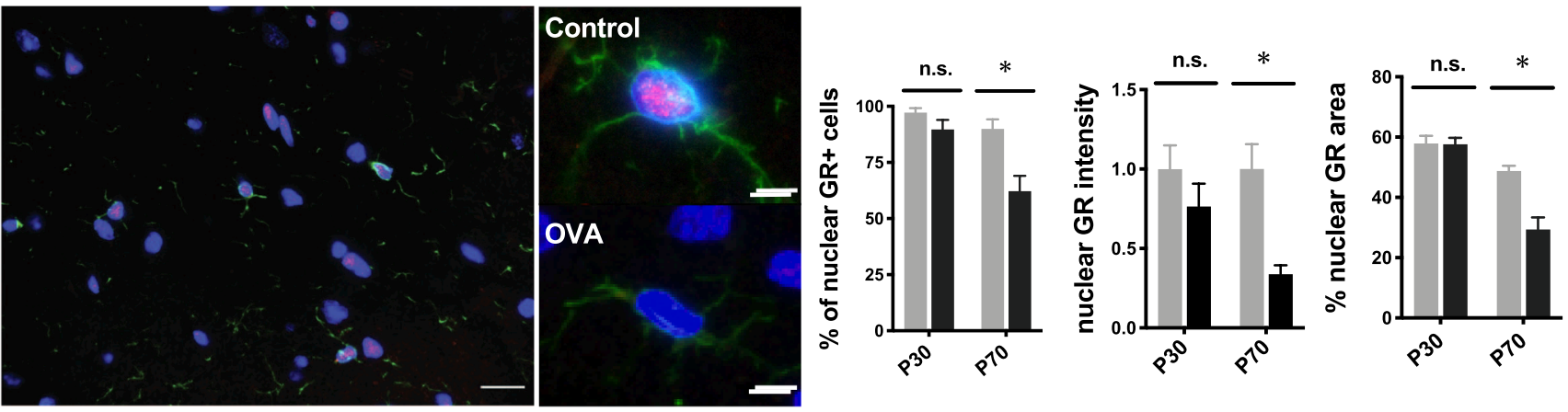

\section{B GR NeuN DAPI}
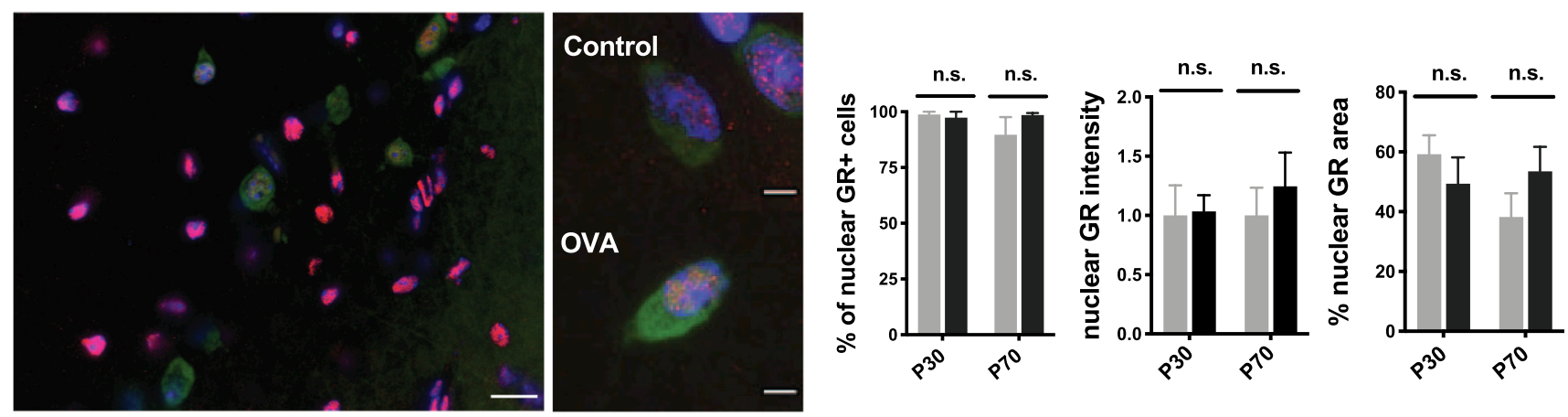

\section{GR S100ß DAPI}
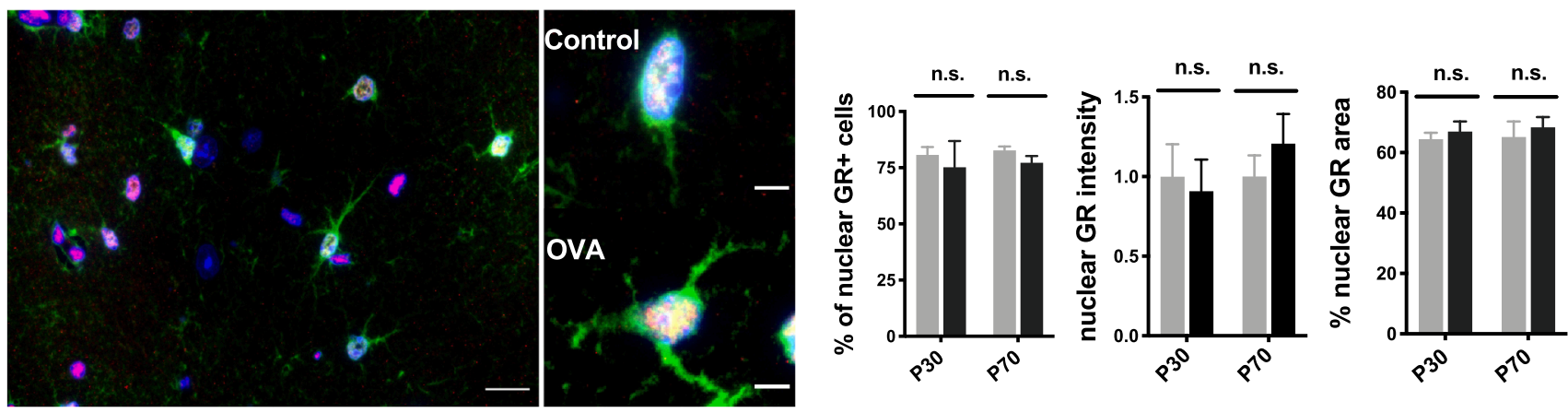

Fig. 9. Effects of short- and long-term allergic airway inflammation from the early postnatal period to puberty and adolescence on glucocorticoid receptor (GR) localization in hippocampal cells. Representative immunofluorescence images of GR, 4', 6-diamidino-2-phenylindole (DAPI), and staining of (A) Iba-1 for microglia, (B) Neu-N for neurons, and (C) S100ß for astrocytes on P70. High-magnification images are shown in the right column. Graphs show the percentage of nuclear GR positive-stained cells (left), ratios of mean of nuclear GR staining intensity (versus control group) (middle), and percentage of nuclear GR-stained areas (right), in each cell type in the CA1-3 of the hippocampus. Analysis of nuclear GR positive-stained cells: microglia, $n=4-5 /$ group for P30, $n=5-6 /$ group for P70; neurons, $n=$ 5/group for P30, $n=4-6 /$ group for P70; astrocytes, $n=5-6 /$ group for P30, $n=4-6 /$ group for P70. Analysis of nuclear GR intensity (versus control group): microglia, $n=5$ /group for P30 and P70; neurons, $n=5$ /group for P30 and P70; astrocytes, $n=5 /$ group for P30 and P70. Analysis of nuclear GR area: microglia, $n=$ 4/group for P30, $n=5$ /group for P70; neurons, $n=4-5$ /group for P30, $n=5-6$ /group for P70; astrocytes, $n=4-5$ /group for P30, $n=5-6 /$ group for P70. Scale bars: left column, $20 \mu \mathrm{m}$; right column, $5 \mu \mathrm{m}$. All values are shown as means \pm SEM. * $p<0.05$ (Mann-Whitney $U$ test).

The upregulation of Th2 cytokines, such as IL-4 and IL-5, and proinflammatory cytokines, such as IL-6, TNF $\alpha$, and GM-CSF, in the OVA mouse BALF compared with control mouse BALF on P30 indicated robust allergic airway inflammation was induced by short-term OVA exposure. However, on P70, the upregulation of Th2 cytokines subsided and proinflammatory cytokines, such as IL-2 and IFN- $\gamma$, were suppressed; only IL-17 was upregulated in the OVA group compared with the control group. These shifts in BALF cytokine profiles suggest Th2- to Th17-mediated airway inflammation in our asthma model was induced by acute-to-chronic allergen exposure. This is consistent with previous reports showing IL-17-mediated airway inflammation was predominant in chronic asthma models associated with increased IL-17 
levels, whereas classic Th2 cytokines such as IL-4 and IL-5 that are elevated in acute asthma models were suppressed in the BALF (Kim et al., 2013; Wang and Wills-Karp, 2011; Zhou et al., 2017). Because IL17 downregulates Th2 and Th1 responses (Ajendra et al., 2020; Ta et al., 2019), the decrease in IFN- $\gamma$ in the BALF on P70 may be caused by overactive Th17 cells in chronic airway inflammation. However, only mild changes were observed in plasma cytokine profiles in our model, which may be explained by asthma having localized airway inflammation rather than systemic inflammation (Bjermer, 2007) The upregulation of IL-5 and IL- 6 in the OVA group on P30 was compatible with acute allergic inflammation and previous reports showing increased plasma IL-5 and IL-13 in an acute asthma model (Klein et al., 2016). On P70, only IFN- $\gamma$ was significantly higher in the OVA group than in the control group $(p<0.05)$. Although the roles of IFN- $\gamma$ in asthma are unclear, airway IFN- $\gamma{ }^{+} \mathrm{CD} 4{ }^{+}$Th1 cells, which are more abundant in severe asthma than mild-to-moderate asthma (Lambrecht et al., 2019; Raundhal et al., 2015), might be involved in chronic asthma models (Kim et al., 2013; Kumar et al., 2006). Overall, the BALF and plasma cytokine profiles in our models on P30 and P70 were compatible with acute and chronic asthma inflammation, respectively.

In mice, synaptogenesis occurs vigorously during the first 3 weeks after birth, peaking at postnatal week 2 . Synaptic pruning by microglia follows synaptogenesis, peaking around postnatal weeks 3-4 (Supplementary Fig. 5) (Jawaid et al., 2018; Semple et al., 2013). At the peak of synaptic pruning, synaptic engulfment by microglia appeared to be decreased in the OVA group compared with controls, as shown by decreased PSD95-occupied areas in Iba-1-positive microglia and the reduced expression of the engulfment-related gene set (Ayata et al., 2018) in the OVA group. Downregulation of the sensome gene set, which encodes proteins involved in microglial process sensing (Hickman et al., 2013) may also be associated with decreased synaptic engulfment by microglia on P30. Conversely, on P70, PSD95-occupied areas in Iba-1positive microglia recovered to control levels and engulfment-related gene expression was correspondingly upregulated whereas CD68positive lysosome areas in Iba-1-positive microglia were decreased, which suggests reduced lysosomal protein expression in the OVA group. Reduced digestion of engulfed particles in dystrophic microglia was reported in aged rhesus monkeys and a mouse model of Alzheimer's disease (Floden and Combs, 2011; Peters et al., 1991). In the OVA group, a non-statistically significant increase in the postsynaptic density markers such as PSD95 and SynGap as well as GluA2 and GLuN2B glutamate receptors was observed on P30 and became highly significant on P70, which suggests excess synapses. PSD95 is a major scaffolding molecule for glutamate receptors localized postsynaptically (Keith and El-Husseini, 2008) and is a useful excitatory synapse marker (Chen et al., 2014a). Therefore, an increase of PSD95 and glutamate receptors suggests an excess of excitatory synapses in the long-term allergic airway inflammation model. The involvement of excitatory synapses was also demonstrated in a genetic mouse model of ASD (Isshiki et al., 2014). Given the absence of augmented neurogenesis on P30 and P70 in the OVA group, excitatory synaptic surplus might be partly caused by impaired engulfment and lysosomal digestion of dystrophic microglia on P30 and P70, respectively.

Redundant synapse pruning by microglia is critical for normal neuronal development from the early postnatal period to puberty (Arcuri et al., 2017; Paolicelli et al., 2011). Defective pruning and the resultant excess of weak synapses are thought to be a pathogenic mechanism for ASD, although it is etiologically and pathologically heterogeneous. Notably, in our early postnatal allergen exposure model, reduced sociability and increased repetitive behavior, both of which are core features of ASD (DSM 5; American Psychiatric Association, 2013), were recognized only on P70 when the increase in postsynaptic density markers was marked. The emergence of dystrophic microglia by shortterm allergen exposure was not sufficient to produce ASD-like behavior manifestations on P30. This is in accord with the observation that maternal exposure to diesel exhaust particles increased the numbers of round microglia with thick processes in male offspring on P30, which was insufficient to affect their behavior (Bilbo et al., 2018). Thus, persistent long-term allergen exposure may be important for the induction of behavioral manifestations mediated partly through synaptic surplus caused by persistently dysfunctioning dystrophic microglia.

Few experimental studies have investigated a causative relationship between allergy and ASD-like behavior; however, accumulating evidence suggests a close link between the two conditions (Chen et al., 2014b; Roth et al., 1991; Theoharides et al., 2016). Maternal immune activation (MIA) induced by lipopolysaccharide, a major component of the outer membrane of Gram-negative bacteria, or polyinosinic:polycytidylic acid, a viral mimic, induced ASD-like behavioral changes in offspring (Le Belle et al., 2014; Bilbo et al., 2008). Therefore, maternal allergic asthma induced by sensitization and aerosol exposure to OVA was investigated to demonstrate decreased sociability and increased marble-burying behavior in offspring (Schwartzer et al., 2015). This model exhibited increased serotonin-transporter protein levels in the cortex; however, no pathological basis was examined. Food allergy in mice at puberty induced by sensitization and challenge with OVA was reported to induce high levels of anxiety and increased Fos expression in the paraventricular nucleus of the hypothalamus (Basso et al., 2003). Allergic rhinitis induced in adult mice by OVA sensitization and challenge reduced social interaction and increased anxiety together with the increased mRNA expression of corticotropin-releasing hormone in the prefrontal cortex (Tonelli et al., 2009). Subsequently, it was shown that food allergy induced shortly after weaning reduced social behavior and increased repetitive behavior in mice, in which the expression of a neuronal activation marker, c-Fos, was enhanced in the prefrontal cortex (de Theije et al., 2014). However, the mechanism of neuronal activation and its relationship to behavioral changes remain elusive in these models. Recently, the reduction of Iba-1-positive microglia, particularly major histocompatibility complex class II-positive microglia, was reported in the hippocampus of a mouse lung allergy model induced by sensitization and challenge with pollen, which suggests reduced microglial surveillance and activity (Klein et al., 2016); however, no behavioral change was described. Whole genome bisulfite sequencing to examine DNA methylation and RNA sequencing to examine gene expression in microglia from maternal allergic asthma model offspring showing ASD-like behavioral changes revealed that differentially methylated regions were enriched for immune signaling pathways and important microglial developmental transcription factor binding motifs. Furthermore, differentially expressed genes that significantly overlapped with genes with altered expression in the human ASD cortex were involved in controlling microglial sensitivity to the environment and shaping neuronal connections in the developing brain (Vogel Ciernia et al., 2018). These previous studies support a role for microglia in the pathogenesis of ASD. These studies did not examine lung pathology; however, the current study performed pathological analyses to confirm asthmatic inflammation in the lung.

To the best of our knowledge, this is the first study to successfully demonstrate the emergence of dystrophic microglia, excitatory synapse marker excess, and ASD-like behavior by early postnatal long-term allergic airway inflammation. It is plausible that impaired synaptic pruning by dystrophic microglia with augmented senescence-related gene expression might lead to synaptic excess and result in ASD-like behavioral deficits. In the well-established MIA model of ASD, various deficits of microglia were also reported (Bergdolt and Dunaevsky, 2019), including altered microglia migration (Zhang et al., 2018), increased immature microglia presenting with an amoeboid morphology (Zhang et al., 2018), and reduced phagocytic activity leading to abnormal pruning (Bergdolt and Dunaevsky, 2019). Thus, our postnatal long-term airway allergy model captures these features of microglia in the MIA model. In addition, increased excitatory glutamatergic synapse transmission (Li et al., 2018) and reduced GABAergic inhibitory synapse transmission (Canetta et al., 2016) were observed in the MIA model-interestingly, the increase in excitatory glutamatergic synapse 
transmission is consistent with the increase in excitatory AMPA and NMDA receptors in our model. Therefore, our study underscores the importance of sustained allergic insults from the early postnatal period to adolescence to induce ASD-like behavioral changes and the relevant pathological changes in the brain, even though the mothers do not develop allergy.

Regarding peripheral cytokine-to-brain communication, particularly to brain microglia, increased IFN- $\gamma$ levels in the blood on P70 in our model might activate microglia to cause neuronal damage (Ta et al., 2019). Immune cell signaling molecules, including TNF- $\alpha$, which was elevated in the BALF of our asthma mouse model, have a major impact on vagal afferent terminals in the airway (Chavan et al., 2017; Frasch et al., 2016); therefore, they might activate afferent vagal nerves via cytokine receptors and pathogen-recognition receptors expressed on vagal afferent neurons or chemosensory cells in the associated vagal paraganglia, which might then influence microglia through the cholinergic pathway (Chavan et al., 2017; Frasch et al., 2016). Whether these systemic cytokines affect brain microglia via the blood or locally through vagal nerves requires further study.

Our model showed elevated basal corticosterone levels in the sera and downregulated microglial GR in the hippocampus in the OVA group on P70. The effects of GCs in the brain are mediated by MR and GR, with the latter being more abundant in microglia and responsible for stress (Duque and Munhoz, 2016; van Olst et al., 2018). Long-term exposure to GC leads to compensatory GR downregulation in the hippocampus ( $\mathrm{Hu}$ et al., 2016), a process known as homologous downregulation, which involves a decrease in GR mRNA and protein levels (Wallace et al., 2010). Microglia might be involved in brain GC downmodulation because they express abundant GR and are primary immune effectors in the CNS (Frank et al., 2007). In addition, a small but significant increase of $\mathrm{HSP}^{+}$microglia in the OVA group on P70 suggests that altered microglial GR translocation might occur in a fraction of microglia in addition to decreased GR production by long-term airway inflammation, given that Hsp90 is a cytosolic chaperone protein that mediates GR maturation and translocation (Grad and Picard, 2007). Therefore, it is reasonable to assume that HPA activation associated with long-term allergic airway inflammation induces compensatory GR downmodulation in brain microglia (Desmet and De Bosscher, 2017; Madalena and Lerch, 2017; Wang et al., 1997). In the present study, we only measured serum corticosterone on P30 and P70; therefore, we could not specifically determine when this elevation of serum corticosterone began in our model. However, on P70 we observed increased serum corticosterone and the downmodulation of microglial GR. HPA activation is a rapid response process and repeated HPA activation can downmodulate GR expression in cells expressing GR including microglia (Hu et al., 2016). Therefore, GR downmodulation in microglia in our asthma model might be attributable to an increase in GC by repeated HPA activation by OVA challenge (Desmet et al., 2017; Madalena and Lerch, 2017; Wang et al., 1997).

The involvement of the HPA axis in allergic disease is complex. Antigenic challenge alone led to increased levels of plasma GC in humans with asthma and allergic rhinitis (Kalogeromitros et al., 2007; Schleimer, 2000). Activation of the HPA axis involves the release of corticotropin-releasing hormone by the paraventricular nucleus of the hypothalamus, which stimulates the production of adrenocorticotropic hormone (ACTH) in the anterior lobe of the pituitary gland. Then, ACTH secreted into the peripheral circulation stimulates the adrenal cortex, releasing GC into the blood (Chen and Miller, 2007). However, prolonged exposure to GC downregulates the expression and function of GR, which causes GC resistance and exacerbates asthma. Although HPA activation has also been reported in depression (DeFilippis, 2018), behavioral findings suggestive of depression such as decreased locomotor activity and body weight loss (Silverman and Sternberg, 2012) were not observed in our model.

GC has pro- and anti-inflammatory roles in neuroinflammation. GC can shift microglia to a primed state with the enhanced expression of major histocompatibility complex class II antigens via increased NOD-, LRR- and pyrin domain-containing protein 3 expression (Desmet and De Bosscher, 2017; Frank et al., 2010; Miller et al., 2007). Such primed microglia secrete a variety of proinflammatory cytokines such as TNF- $\alpha$, IL-1 $\beta$, and IL-6 upon subsequent lipopolysaccharide exposure (Desmet and De Bosscher, 2017). Conversely, GC inhibited microglial proliferation and caused shrinkage of microglial somata with decreased inducible nitric oxide synthase activity and acid phosphatase concentrations in vitro (Tanaka et al., 1997). Thus, increased GC due to HPA activation might be partly responsible for the dystrophy and shrunken morphology of microglia in our model.

Although HPA axis activation in ASD remains to be established, it was reported that ASD patients had higher basal levels of ACTH and GC levels in their sera compared with healthy controls, which partly reflects a heightened stress response to the environment (Iwata et al., 2011; Lam et al., 2006; Tordjman et al., 1997). Interestingly, reduced mRNA and protein levels of GR in the frontal gyrus of ASD brains were reported together with the increased mRNA expressions of IL-1 $\beta$ and IFN- $\gamma$ (Patel et al., 2016). Our results in the long-term allergic airway inflammation model exhibiting ASD-like behavior abnormalities are in accord with these findings in humans.

This study had several limitations. First, synapses were not morphometrically counted in the present study. Excitatory and inhibitory synapses should be differentially measured in a future study. Second, proteins related to sensome and senescence gene sets as well as microglial activation markers were not examined by immunohistochemistry and immunoblotting, which should also be done in a future study. Finally, we only studied two time points. A more detailed time course study is warranted to characterize the development of microglial functional changes, synaptic excess, HPA axis activation, and compensatory downregulation of GR in microglia.

In summary, we conclude that early postnatal allergic airway inflammation induces dystrophic morphological changes of microglia, which exhibit defective synaptic pruning, even after short-term allergen exposure. However, only long-term allergen exposure caused excitatory synaptic excess and ASD-like behavioral changes. HPA axis activation and the compensatory downregulation of microglia GR by long-term allergic airway inflammation may also facilitate these changes.

\section{Funding}

This study was supported in part by the Practical Research Project for Rare/Intractable Diseases from the Japan Agency for Medical Research and Development (AMED) (JP20ek019308h0003) and JSPS KAKENHI Grants-in-Aid for Scientific Research (A) (Grant Number JP16H02657 and JP19H01045).

\section{Declaration of Competing Interest}

BS, ET, NY, DK, KI, YN, and HY declare no conflict of interest. RY received honoraria from Biogen Japan, Ono Pharmaceuticals, Teijin Pharma, Novartis Pharma, and Takeda Pharmaceutical. KM received grants and personal fees from AstraZeneca Japan, Boehringer Ingelheim Japan, KYORIN Pharmaceutical, Novartis Pharma, and Sanofi Japan. JK received grants and personal fees from Biogen Japan, Bayer Healthcare, Novartis Pharma, Mitsubishi Tanabe Pharma, Eisai, Sanofi, Nobelpharma, Otsuka Pharmaceutical, Chugai Pharmaceutical Company, and Teijin Pharma.

\section{Acknowledgements}

We appreciate technical assistance from The Research Support Center, Research Center for Human Disease Modeling, Kyushu University Graduate School of Medical Sciences, and data interpretation of allergic asthma by Dr. Satoru Fukuyama and Dr. Keiko Kan-o, Research Institute for Diseases of the Chest, Graduate School of Medical Sciences, 
Kyushu University. We thank J. Ludovic Croxford, PhD, from Edanz Group (https://en-author-services.edanz.com/ac) for editing a draft of this manuscript.

\section{Appendix A. Supplementary data}

Supplementary data to this article can be found online at https://doi. org/10.1016/j.bbi.2021.04.008.

\section{References}

Ajendra, J., Chenery, A.L., Parkinson, J.E., Chan, B.H.K., Pearson, S., Colombo, S.A.P. Boon, L., Grencis, R.K., Sutherland, T.E., Allen, J.E., 2020. IL-17A both initiates, via IFN $\gamma$ suppression, and limits the pulmonary type-2 immune response to nematode infection. Mucosal Immunol. https://doi.org/10.1038/s41385-020-0318-2.

American Psychiatric Association, 2013. American Psychiatric Association: Diagnostic and Statistical Manual of Mental Disorders Fifth Edition, Arlington. 10.1176/appi. books. 9780890425596.744053.

Angoa-Pérez, M., Kane, M.J., Briggs, D.I., Francescutti, D.M., Kuhn, D.M., 2013. Marble burying and nestlet shredding as tests of repetitive, compulsive-like behaviors in mice. J. Vis. Exp. 50978 https://doi.org/10.3791/50978,

Arcuri, C., Mecca, C., Bianchi, R., Giambanco, I., Donato, R., 2017. The pathophysiological role of microglia in dynamic surveillance, phagocytosis and structural remodeling of the developing CNS. Front. Mol. Neurosci. 10.3389/ fnmol.2017.00191.

Ayata, P., Badimon, A., Strasburger, H.J., Duff, M.K., Montgomery, S.E., Loh, Y.H.E., Ebert, A., Pimenova, A.A., Ramirez, B.R., Chan, A.T., Sullivan, J.M., Purushothaman, I., Scarpa, J.R., Goate, A.M., Busslinger, M., Shen, L., Losic, B., Schaefer, A., 2018. Epigenetic regulation of brain region-specific microglia clearance activity. Nat. Neurosci. 21, 1049-1060. https://doi.org/10.1038/s41593-018-01923.

Basso, A.S., Costa Pinto, F.A., Russo, M., Giorgetti Britto, L.R., De Sá-Rocha, L.C., Palermo Neto, J., 2003. Neural correlates of IgE-mediated food allergy. J. Neuroimmunol. https://doi.org/10.1016/S0165-5728(03)00166-8.

Bergdolt, L., Dunaevsky, A., 2019. Brain changes in a maternal immune activation model of neurodevelopmental brain disorders. Prog. Neurobiol. 10.1016/j. pneurobio.2018.12.002.

Bilbo, S.D., Barrientos, R.M., Eads, A.S., Northcutt, A., Watkins, L.R., Rudy, J.W., Maier, S.F., 2008. Early-life infection leads to altered BDNF and IL-1 $\beta$ mRNA expression in rat hippocampus following learning in adulthood. Brain. Behav. Immun. https://doi.org/10.1016/j.bbi.2007.10.003.

Bilbo, S.D., Block, C.L., Bolton, J.L., Hanamsagar, R., Tran, P.K., 2018. Beyond infection Maternal immune activation by environmental factors, microglial development, and relevance for autism spectrum disorders. Exp. Neurol. 10.1016/j. expneurol.2017.07.002

Bjermer, L., 2007. Time for a paradigm shift in asthma treatment: From relieving bronchospasm to controlling systemic inflammation. J. Allergy Clin. Immunol. https://doi.org/10.1016/j.jaci.2007.09.017.

Blatt, G.J., 2012. The neuropathology of autism. Scientifica (Cairo). https://doi.org/ 10.6064/2012/703675.

Canetta, S., Bolkan, S., Padilla-Coreano, N., Song, L.J., Sahn, R., Harrison, N.L., Gordon, J.A., Brown, A., Kellendonk, C., 2016. Maternal immune activation leads to selective functional deficits in offspring parvalbumin interneurons. Mol. Psychiatry. 10.1038/ mp.2015.222.

Chavan, S.S., Pavlov, V.A., Tracey, K.J., 2017. Mechanisms and therapeutic relevance of neuro-immune communication. Immunity. https://doi.org/10.1016/j. immuni.2017.06.008.

Chen, E., Miller, G.E., 2007. Stress and inflammation in exacerbations of asthma. Brain. Behav. Immun. https://doi.org/10.1016/j.bbi.2007.03.009.

Chen, J., Yu, S., Fu, Y., Li, X., 2014a. Synaptic proteins and receptors defects in autism spectrum disorders. Front. Cell. Neurosci. 8, 1-13. https://doi.org/10.3389/ fncel.2014.00276.

Chen, M.H., Su, T.P., Chen, Y.S., Hsu, J.W., Huang, K.L., Chang, W.H., Chen, T.J., Pan, T. L., Bai, Y.M., 2014b. Is atopy in early childhood a risk factor for ADHD and ASD? A longitudinal study. J Psychosom Res 77, 316-321.

DeFilippis, M., 2018. Depression in children and adolescents with autism spectrum disorder. Children. https://doi.org/10.3390/children5090112.

Desmet, S.J., De Bosscher, K., 2017. Glucocorticoid receptors: Finding the middle ground. J. Clin. Invest. 127, 1136-1145. https://doi.org/10.1172/JCI88886.

De Theije, C.G.M., Wu, J., Koelink, P.J., Korte-Bouws, G.A.H., Borre, Y., Kas, M.J.H., Lopes da Silva, S., Korte, S.M., Olivier, B., Garssen, J., Kraneveld, A.D., 2014. Autistic-like behavioural and neurochemical changes in a mouse model of food allergy. Behav. Brain Res. 10.1016/j.bbr.2013.12.008.

Duque, E. de A., Munhoz, C.D., 2016. The pro-inflammatory effects of glucocorticoids in the brain. Front. Endocrinol. (Lausanne) 7, 1-7. https://doi.org/10.3389/ fendo.2016.00078.

Floden, A.M., Combs, C.K., 2011. Microglia demonstrate age-dependent interaction with amyloid- $\beta$ fibrils. J. Alzheimer's Dis. https://doi.org/10.3233/JAD-2011-101014.

Frank, M.G., Baratta, M.V., Sprunger, D.B., Watkins, L.R., Maier, S.F., 2007. Microglia serve as a neuroimmune substrate for stress-induced potentiation of CNS proinflammatory cytokine responses. Brain. Behav. Immun. https://doi.org/10.1016/j. bbi.2006.03.005.
Frank, M.G., Miguel, Z.D., Watkins, L.R., Maier, S.F., 2010. Prior exposure to glucocorticoids sensitizes the neuroinflammatory and peripheral inflammatory responses to E. coli lipopolysaccharide. Brain. Behav. Immun. https://doi.org/ 10.1016/j.bbi.2009.07.008.

Frasch, M.G., Szynkaruk, M., Prout, A.P., Nygard, K., Cao, M., Veldhuizen, R., Hammond, R., Richardson, B.S., 2016. Decreased neuroinflammation correlates to higher vagus nerve activity fluctuations in near-term ovine fetuses: A case for the afferent cholinergic anti-inflammatory pathway? J. Neuroinflamm. https://doi.org/ 10.1186/s12974-016-0567-x.

Germundson, D.L., Smith, N.A., Vendsel, L.P., Kelsch, A.V., Combs, C.K., NagamotoCombs, K., 2018. Oral sensitization to whey proteins induces age- and sex-dependent behavioral abnormality and neuroinflammatory responses in a mouse model of food allergy: A potential role of mast cells. J. Neuroinflammation. https://doi.org/ 10.1186/s12974-018-1146-0.

Gong, S., Miao, Y.L., Jiao, G.Z., Sun, M.J., Li, H., Lin, J., Luo, M.J., Tan, J.H., 2015. Dynamics and correlation of serum cortisol and corticosterone under different physiological or stressful conditions in mice. PLoS ONE. https://doi.org/10.1371/ journal.pone.0117503.

Grad, I., Picard, D., 2007. The glucocorticoid responses are shaped by molecular chaperones. Mol. Cell. Endocrinol. 10.1016/j.mce.2007.05.018.

Guo, R.B., Sun, P.L., Zhao, A.P., Gu, J., Ding, X., Qi, J., Sun, X.L., Hu, G., 2013. Chronic asthma results in cognitive dysfunction in immature mice. Exp Neurol 247, 209-217. https://doi.org/10.1016/j.expneurol.2013.04.008.

Gyoneva, S., Hosur, R., Gosselin, D., Zhang, B., Ouyang, Z., Cotleur, A.C., Peterson, M., Allaire, N., Challa, R., Cullen, P., Roberts, C., Miao, K., Reynolds, T.L., Glass, C.K. Burkly, L., Ransohoff, R.M., 2019. Cx3cr1-deficient microglia exhibit a premature aging transcriptome. Life Sci. Alliance 2, 1-16. 10.26508/lsa.201900453.

Hamada, K., Goldsmith, C.A., Kobzik, L., 1999. Increased airway hyperresponsiveness and inflammation in a juvenile mouse model of asthma exposed to air-pollutant aerosol. J. Toxicol. Environ. Heal. - Part A. https://doi.org/10.1080/ 009841099157340.

Heindl, S., Gesierich, B., Benakis, C., Llovera, G., Duering, M., Liesz, A., 2018. Automated morphological analysis of microglia after stroke. Front. Cell. Neurosci. 12, 1-11. https://doi.org/10.3389/fncel.2018.00106.

Hickman, S.E., Kingery, N.D., Ohsumi, T.K., Borowsky, M.L., Wang, L.C., Means, T.K., El Khoury, J., 2013. The microglial sensome revealed by direct RNA sequencing. Nat. Neurosci. 16, 1896-1905. https://doi.org/10.1038/nn.3554.

Hu, W., Zhang, Y., Wu, W., Yin, Y., Huang, D., Wang, Y., Li, Weiping, Li, Weizu, 2016. Chronic glucocorticoids exposure enhances neurodegeneration in the frontal cortex and hippocampus via NLRP-1 inflammasome activation in male mice. Brain. Behav. Immun. https://doi.org/10.1016/j.bbi.2015.09.019.

Isshiki, M., Tanaka, S., Kuriu, T., Tabuchi, K., Takumi, T., Okabe, S., 2014. Enhanced synapse remodelling as a common phenotype in mouse models of autism. Nat. Commun. 10.1038/ncomms5742.

Iwata, K., Matsuzaki, H., Miyachi, T., Shimmura, C., Suda, S., Tsuchiya, K.J., Matsumoto, K., Suzuki, K., Iwata, Y., Nakamura, K., Tsujii, M., Sugiyama, T., Sato, K., Mori, N., 2011. Investigation of the serum levels of anterior pituitary hormones in male children with autism. Mol. Autism. 10.1186/2040-2392-2-16.

Jawaid, S., Kidd, G.J., Wang, J., Swetlik, C., Dutta, R., Trapp, B.D., 2018. Alterations in CA1 hippocampal synapses in a mouse model of fragile X syndrome. Glia. https:// doi.org/10.1002/glia.23284.

Kalogeromitros, D., Syrigou, E.K.I., Makris, M., Kempuraj, D., Stavrianeas, N.G., Vasiadi, M., Theoharides, T.C., 2007. Nasal provocation of patients with allergic rhinitis and the hypothalamic-pituitary-adrenal axis. Ann. Allergy, Asthma Immunol. 10.1016/ S1081-1206(10)60717-X.

Keith, D., El-Husseini, A., 2008. Excitation control: Balancing PSD-95 function at the synapse. Front. Mol. Neurosci. 10.3389/neuro.02.004.2008.

Kim, M.S., Cho, K.A., Cho, Y.J., Woo, S.Y., 2013. Effects of interleukin-9 blockade on chronic airway inflammation in murine asthma models. Allergy, Asthma Immunol. Res. 10.4168/aair.2013.5.4.197.

Klein, B., Mrowetz, H., Thalhamer, J., Scheiblhofer, S., Weiss, R., Aigner, L., 2016. Allergy enhances neurogenesis and modulates microglial activation in the hippocampus. Front. Cell. Neurosci. 10.3389/fncel.2016.00169.

Kumar, R.K., Webb, D.C., Herbert, C., Foster, P.S., 2006. Interferon- $\gamma$ as a possible target in chronic asthma. Inflamm. Allergy - Drug Targets. 10.2174/ 187152806779010909.

Kumar, R.K., Herbert, C., Foster, P.S., 2008. The "classical" ovalbumin challenge model of asthma in mice. Curr. Drug Targets. 10.2174/138945008784533561.

Lam, K.S.L., Aman, M.G., Arnold, L.E., 2006. Neurochemical correlates of autistic disorder: A review of the literature. Res. Dev. Disabil. https://doi.org/10.1016/j. ridd.2005.03.003.

Lambrecht, B.N., Hammad, H., Fahy, J.V., 2019. The cytokines of asthma. Immunity. https://doi.org/10.1016/j.immuni.2019.03.018.

Le Belle, J.E., Sperry, J., Ngo, A., Ghochani, Y., Laks, D.R., López-Aranda, M., Silva, A.J., Kornblum, H.I., 2014. Maternal inflammation contributes to brain overgrowth and autism-associated behaviors through altered redox signaling in stem and progenitor cells. Stem Cell Rep. https://doi.org/10.1016/j.stemcr.2014.09.004.

Lee, A.S., Azmitia, E.C., Whitaker-Azmitia, P.M., 2017. Developmental microglial priming in postmortem autism spectrum disorder temporal cortex. Brain. Behav. Immun. https://doi.org/10.1016/j.bbi.2017.01.019.

Lee, M.S., Kim, Y.H., Park, W.S., Park, O.K., Kwon, S.H., Hong, K.S., Rhim, H., Shim, I., Morita, K., Wong, D.L., Patel, P.D., Lyons, D.M., Schatzberg, A.F., Her, S., 2016. Temporal variability of glucocorticoid receptor activity is functionally important for the therapeutic action of fluoxetine in the hippocampus. Mol. Psychiatry. 10.1038/ mp.2014.137. 
Lenz, K.M., Pickett, L.A., Wright, C.L., Galan, A., McCarthy, M.M., 2019. Prenatal Allergen Exposure Perturbs Sexual Differentiation and Programs Lifelong Changes in Adult Social and Sexual Behavior. Sci. Rep. 10.1038/s41598-019-41258-2.

Li, Y., Missig, G., Finger, B.C., Landino, S.M., Alexander, A.J., Mokler, E.L., Robbins, J.O., Manasian, Y., Kim, W., Kim, K.S., McDougle, C.J., Carlezon Jr., W.A., Bolshakov, V. Y., 2018. Maternal and early postnatal immune activation produce dissociable effects on neurotransmission in mPFC-amygdala circuits. J. Neurosci. 38 3358-3372.

Lo, S.C., Scearce-Levie, K., Sheng, M., 2016. Characterization of Social Behaviors in caspase-3 deficient mice. Sci. Rep. 10.1038/srep18335.

Lu, C., Zhang, B., Xu, T., Zhang, W., Bai, B., Xiao, Z., Wu, L., Liang, G., Zhang, Y., Dai, Y., 2019. Piperlongumine reduces ovalbumin-induced asthma and airway inflammation by regulating nuclear factor-кB activation. Int. J. Mol. Med. 10.3892/ ijmm.2019.4322.

Maatouk, L., Compagnion, A.C., Sauvage, M.A.C. De, Bemelmans, A.P., Leclere-Turbant, S., Cirotteau, V., Tohme, M., Beke, A., Trichet, M., Bazin, V., Trawick, B.N., Ransohoff, R.M., Tronche, F., Manoury, B., Vyas, S., 2018. TLR9 activation via microglial glucocorticoid receptors contributes to degeneration of midbrain dopamine neurons. Nat. Commun. 10.1038/s41467-018-04569-y.

Maatouk, L., Yi, C., Carrillo-de Sauvage, M.A., Compagnion, A.C., Hunot, S., Ezan, P., Hirsch, E.C., Koulakoff, A., Pfrieger, F.W., Tronche, F., Leybaert, L., Giaume, C., Vyas, S., 2019. Glucocorticoid receptor in astrocytes regulates midbrain dopamine neurodegeneration through connexin hemichannel activity. Cell Death Differ. https://doi.org/10.1038/s41418-018-0150-3.

Madalena, K.M., Lerch, J.K., 2017. The effect of glucocorticoid and glucocorticoid receptor interactions on brain, spinal cord, and glial cell plasticity. Neural Plast. 2017 https://doi.org/10.1155/2017/8640970.

Malkova, N.V., Yu, C.Z., Hsiao, E.Y., Moore, M.J., Patterson, P.H., 2012. Maternal immune activation yields offspring displaying mouse versions of the three core symptoms of autism. Brain. Behav. Immun. https://doi.org/10.1016/j. bbi.2012.01.011.

Martín-de-Saavedra, M.D., Budni, J., Cunha, M.P., Gómez-Rangel, V., Lorrio, S., del Barrio, L., Lastres-Becker, I., Parada, E., Tordera, R.M., Rodrigues, A.L.S. Cuadrado, A., López, M.G., 2013. Nrf2 participates in depressive disorders through an anti-inflammatory mechanism. Psychoneuroendocrinology 38. https://doi.org/ 10.1016/j.psyneuen.2013.03.020.

Miller, G.E., Chen, E., Zhou, E.S., 2007. If it goes up, must it come down? Chronic stress and the hypothalamic-pituitary-adrenocortical axis in humans. Psychol. Bull. https://doi.org/10.1037/0033-2909.133.1.25.

Myou, S., Leff, A.R., Myo, S., Boetticher, E., Tong, J., Meliton, A.Y., Liu, J., Munoz, N.M., Zhu, X., 2003. Blockade of Inflammation and Airway Hyperresponsiveness in Immune-sensitized Mice by Dominant-Negative Phosphoinositide 3-Kinase-TAT. J. Exp. Med. https://doi.org/10.1084/jem.20030298.

Paolicelli, R.C., Bolasco, G., Pagani, F., Maggi, L., Scianni, M., Panzanelli, P., Giustetto, M., Ferreira, T.A., Guiducci, E., Dumas, L., Ragozzino, D., Gross, C.T. 2011. Synaptic pruning by microglia is necessary for normal brain development. Science 80-, ). https://doi.org/10.1126/science.1202529.

Paolicelli, R.C., Gross, C.T., 2012. Microglia in development: Linking brain wiring to brain environment. Neuron Glia Biol. https://doi.org/10.1017/ S1740925X12000105.

Patel, N., Crider, A., Pandya, C.D., Ahmed, A.O., Pillai, A., 2016. Altered mRNA Levels of Glucocorticoid Receptor, Mineralocorticoid Receptor, and Co-Chaperones (FKBP5 and PTGES3) in the Middle Frontal Gyrus of Autism Spectrum Disorder Subjects. Mol. Neurobiol. 53, 2090-2099. https://doi.org/10.1007/s12035-015-9178-2.

Patel, S., Dale, R.C., Rose, D., Heath, B., Nordahl, C.W., Rogers, S., Guastella, A.J., Ashwood, P., 2020. Maternal immune conditions are increased in males with autism spectrum disorders and are associated with behavioural and emotional but not cognitive co-morbidity. Transl. Psychiatry. 10.1038/s41398-020-00976-2.

Petanjek, Z., Judaš, M., Šimić, G., Rašin, M.R., Uylings, H.B.M., Rakic, P., Kostović, I., 2011. Extraordinary neoteny of synaptic spines in the human prefrontal cortex. Proc. Natl. Acad. Sci. U. S. A. 10.1073/pnas.1105108108

Peters, A., Josephson, K., Vincent, S.L., 1991. Effects of aging on the neuroglial cells and pericytes within area 17 of the rhesus monkey cerebral cortex. Anat. Rec. 10.1002/ ar.1092290311.

Prut, L., Belzung, C., 2003. The open field as a paradigm to measure the effects of drugs on anxiety-like behaviors: A review. Eur. J. Pharmacol. 463, 3-33. https://doi.org/ 10.1016/S0014-2999(03)01272-X.

Raundhal, M., Morse, C., Khare, A., Oriss, T., Milosevic, J., Trudeau, J., Huff, R., Pilewski, J., Holguin, F., Kolls, J., Wenzel, S., Ray, P., Ray, A., 2015. High IFN- $\gamma$ and low SLPI mark severe asthma in mice and humans. J. Clin. Invest.

Raymond, G.V., Bauman, M.L., Kemper, T.L., 1995. Hippocampus in autism: a Golgi analysis. Acta Neuropathol. https://doi.org/10.1007/s004010050401.

Reemst, K., Noctor, S.C., Lucassen, P.J., Hol, E.M., 2016. The indispensable roles of microglia and astrocytes during brain development. Front. Hum. Neurosci. 10.3389/ fnhum.2016.00566.

Robillard, K.N., Lee, K.M., Chiu, K.B., MacLean, A.G., 2016. Glial cell morphological and density changes through the lifespan of rhesus macaques. Brain. Behav. Immun. https://doi.org/10.1016/j.bbi.2016.01.006.
Roth, N., Beyreiss, J., Schlenzka, K., Beyer, H., 1991. Coincidence of attention deficit disorder and atopic disorders in children: Empirical findings and hypothetical background. J. Abnorm. Child Psychol. https://doi.org/10.1007/BF00910561.

Saglani, S., Mathie, S.A., Gregory, L.G., Bell, M.J., Bush, A., Lloyd, C.M., 2009. Pathophysiological features of asthma develop in parallel in house dust mite-exposed neonatal mice. Am. J. Respir. Cell Mol. Biol. https://doi.org/10.1165/rcmb.200803960C.

Sanagi, T., Sasaki, T., Nakagaki, K., Minamimoto, T., Kohsaka, S., Ichinohe, N., 2019. Segmented Iba1-Positive Processes of Microglia in Autism Model Marmosets. Front. Cell. Neurosci. 13, 1-8. https://doi.org/10.3389/fncel.2019.00344.

Schleimer, R.P., 2000. Interactions between the hypothalamic-pituitary-adrenal axis and allergic inflammation. J. Allergy Clin. Immunol. https://doi.org/10.1067/ mai.2000.110162.

Schwartzer, J.J., Careaga, M., Chang, C., Onore, C.E., Ashwood, P., 2015. Allergic fetal priming leads to developmental, behavioral and neurobiological changes in mice. Transl. Psychiatry. 10.1038/tp.2015.40.

Semple, B.D., Blomgren, K., Gimlin, K., Ferriero, D.M., Noble-Haeusslein, L.J., 2013. Brain development in rodents and humans: Identifying benchmarks of maturation and vulnerability to injury across species. Prog. Neurobiol. 10.1016/j. pneurobio.2013.04.001.

Silverman, M.N., Sternberg, E.M., 2012. Glucocorticoid regulation of inflammation and its functional correlates: From HPA axis to glucocorticoid receptor dysfunction. Ann. N. Y. Acad. Sci. 10.1111/j.1749-6632.2012.06633.x.

Spittau, B., 2017. Aging microglia-phenotypes, functions and implications for age-related neurodegenerative diseases. Front. Aging Neurosci. 10.3389/fnagi.2017.00194.

Subramanian, A., Tamayo, P., Mootha, V.K., Mukherjee, S., Ebert, B.L., Gillette, M.A., Paulovich, A., Pomeroy, S.L., Golub, T.R., Lander, E.S., Mesirov, J.P., 2005. Gene set enrichment analysis: A knowledge-based approach for interpreting genome-wide expression profiles. Proc. Natl. Acad. Sci. U. S. A. 10.1073/pnas.0506580102.

Ta, T.T., Dikmen, H.O., Schilling, S., Chausse, B., Lewen, A., Hollnagel, J.O., Kann, O., 2019. Priming of microglia with IFN- $\gamma$ slows neuronal gamma oscillations in situ. Proc. Natl. Acad. Sci. U. S. A. 10.1073/pnas.1813562116.

Tanaka, J., Fujita, H., Matsuda, S., Toku, K., Sakanaka, M., Maeda, N., 1997. Glucocorticoid- and mineralocorticoid receptors in microglial cells: The two receptors mediate differential effects of corticosteroids. Glia. https://doi.org/ 10.1002/(SICI)1098-1136(199705)20:123::AID-GLIA33.0.CO;2-6.

Theoharides, T.C., Tsilioni, I., Patel, A.B., Doyle, R., 2016. Atopic diseases and inflammation of the brain in the pathogenesis of autism spectrum disorders. Transl. Psychiatry. 10.1038/tp.2016.77.

Tonelli, L.H., Katz, M., Kovacsics, C.E., Gould, T.D., Joppy, B., Hoshino, A., Hoffman, G., Komarow, H., Postolache, T.T., 2009. Allergic rhinitis induces anxiety-like behavior and altered social interaction in rodents. Brain. Behav. Immun. 23, 784-793. https://doi.org/10.1016/j.bbi.2009.02.017.

Tordjman, S., McBride, P.A., Hertzig, M.E., Snow, M.E., Thompson, S.M., Cohen, D.J. Andersen, G.M., 1997. Plasma $\beta$-endorphin, adrenocorticotropin hormone, and cortisol in autism. J. Child Psychol. Psychiatry Allied Discip. https://doi.org/ 10.1111/j.1469-7610.1997.tb01697.x.

van Olst, L., Bielefeld, P., Fitzsimons, C.P., de Vries, H.E., Schouten, M., 2018. Glucocorticoid-mediated modulation of morphological changes associated with aging in microglia. Aging Cell. https://doi.org/10.1111/acel.12790.

Vogel Ciernia, A., Careaga, M., LaSalle, J.M., Ashwood, P., 2018. Microglia from offspring of dams with allergic asthma exhibit epigenomic alterations in genes dysregulated in autism. Glia. https://doi.org/10.1002/glia.23261.

Wallace, A.D., Cao, Y., Chandramouleeswaran, S., Cidlowski, J.A., 2010. Lysine 419 targets human glucocorticoid receptor for proteasome degradation. Steroids 75 (12), $1016-1023$.

Wang, P.S., Lo, M.J., Kau, M.M., 1997. Glucocorticoids and aging. J. Formos. Med. Assoc. https://doi.org/10.1159/000061414.

Wang, Y.H., Wills-Karp, M., 2011. The potential role of interleukin-17 in severe asthma. Curr. Allergy Asthma Rep. 10.1007/s11882-011-0210-y.

Xie, J.F., Shao, Y.F., Wang, H.L., Wang, C., Cui, G.F., Kong, X.P., Wang, L.X., Chen, Y.N., Cong, C.Y., Chen, H.L., Hou, Y.P., 2018. Neuropeptide s counteracts paradoxical sleep deprivation-induced anxiety-like behavior and sleep disturbances. Front. Cell. Neurosci. 10.3389/fncel.2018.00064.

Yamasaki, R., Fujii, T., Wang, B., Masaki, K., Kido, M.A., Yoshida, M., Matsushita, T., Kira, J.I., 2016. Allergic inflammation leads to neuropathic pain via glial cell activation. J. Neurosci. https://doi.org/10.1523/JNEUROSCI.1981-16.2016.

Zhan, Y., Paolicelli, R.C., Sforazzini, F., Weinhard, L., Bolasco, G., Pagani, F. Vyssotski, A.L., Bifone, A., Gozzi, A., Ragozzino, D., Gross, C.T., 2014. Deficient neuron-microglia signaling results in impaired functional brain connectivity and social behavior. Nat. Neurosci. 17, 400-406. https://doi.org/10.1038/nn.3641.

Zhang, J., Jing, Y., Zhang, H., Bilkey, D.K., Liu, P., 2018. Maternal immune activation altered microglial immunoreactivity in the brain of postnatal day 2 rat offspring. Synapse e22072.

Zhou, T., Huang, X., Zhou, Y., Ma, J., Zhou, M., Liu, Y., Xiao, L., Yuan, J., Xie, J., Chen, W., 2017. Associations between Th17-related inflammatory cytokines and asthma in adults: A Case-Control Study. Sci. Rep. 10.1038/s41598-017-15570-8. 